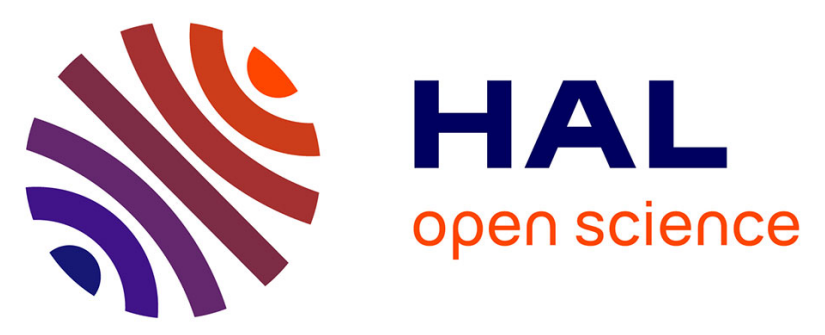

\title{
Magnetometric resistivity: a new approach and its application to the detection of preferential flow paths in mine waste rock dumps
}

\author{
Mike Jessop, Abderrahim Jardani, André Revil, Val Kofoed
}

\section{To cite this version:}

Mike Jessop, Abderrahim Jardani, André Revil, Val Kofoed. Magnetometric resistivity: a new approach and its application to the detection of preferential flow paths in mine waste rock dumps. Geophysical Journal International, 2018, 215 (1), pp.222-239. 10.1093/gji/ggy275 . hal-02324208

\author{
HAL Id: hal-02324208 \\ https://hal.science/hal-02324208
}

Submitted on 21 Oct 2019

HAL is a multi-disciplinary open access archive for the deposit and dissemination of scientific research documents, whether they are published or not. The documents may come from teaching and research institutions in France or abroad, or from public or private research centers.
L'archive ouverte pluridisciplinaire HAL, est destinée au dépôt et à la diffusion de documents scientifiques de niveau recherche, publiés ou non, émanant des établissements d'enseignement et de recherche français ou étrangers, des laboratoires publics ou privés. 


\title{
Magnetometric resistivity: a new approach and its application to the detection of preferential flow paths in mine waste rock dumps
}

\author{
M. Jessop, ${ }^{1}$ A. Jardani, ${ }^{2}$ A. Revil ${ }^{3}$ and V. Kofoed ${ }^{1}$ \\ ${ }^{1}$ Willowstick Technologies, LLC; 132 East 13065 South, Draper, UT 84020, USA \\ ${ }^{2}$ Université de Rouen, M2C, UMR 6143, CNRS, Morphodynamique Continentale et Côtière, Mont Saint Aignan, France \\ ${ }^{3}$ Université Savoie Mont Blanc, CNRS, IRD, IFSTTAR, ISTerre, F-73000 Chambéry,France.E-mail: andre.revil@univ-smb.fr
}

Accepted 2018 July 5. Received 2018 July 1; in original form 2018 May 17

\begin{abstract}
SUMMAR Y
The injection of a low frequency electrical current in the ground between two electrodes A and $B$ generates a magnetic field that can be measured at the ground surface with sensitive magnetic sensors. The map of the magnetic field, measured at the frequency of the injected current, can be used to determine the paths of the current through the ground. When the current is channelled along preferential conductive paths, the MagnetoMetric Resistivity (MMR) method can be used to detect these paths. Conductive current paths can be associated with preferential flow paths of groundwater when the two electrodes A and B are in the direction of the flow and when the flow path is highly electrically conductive with respect to the background. We first review the background equations for the magnetic field in MMR. Then, we provide the kernel of the problem using Biot and Savart law to connect the components of the observed magnetic field to the current density distribution. We also develop a simple approach to invert the magnetic field in terms of electrical current paths. To illustrate how the method works, we develop five synthetic models to test the sensitivity of the method to the properties of the conductive targets channelling the electrical current. The targets are characterized by different shapes, sizes, depths, and conductivity contrasts with the background. Then, we proceed with a case study for which the MMR method is used to identify and map preferential groundwater flow paths by-passing a mine waste rock dump drainage collection trench into the tailings pond. In this case, the conductivity of the flow paths is much stronger than the background conductivity due to the high mineralization of the ground water along these paths. The method underlines the 3-D architecture of these flow paths.
\end{abstract}

Key words: Electrical properties; Hydrogeophysics; Electromagnetic theory; Magnetic anomalies: modelling and interpretation; Tomography.

\section{INTRODUCTION}

The Direct Current (DC) conductivity/resistivity method is a wellknown geophysical technique able to image the low-frequency electrical conductivity distribution of the ground (e.g. Dey \& Morrison 1979; Daily et al. 2004). This method is widely used in hydrogeophysics for a variety of applications (e.g. Revil et al. 2012; Binley et al. 2015). A less known method is the MagnetoMetric Resistivity (MMR) method. With MMR, a low-frequency electrical current (frequency $<1 \mathrm{kHz}$ ) is injected in the ground, between two electrodes A and B and the resulting magnetic field is measured. This method was initially proposed by Jakosky (1933). It was later refined by Stephanescu in Romania (Stefanescu \& Nabighian 1962; Nabighian 1991a,b) with the purpose of determining the electrical conductivity of the Earth (Edwards 1974). Indeed, MMR measurements are sensitive to the gradient of the electrical conductivity (or resistivity) of the ground and offer measurements that are complementary to the classical DC conductivity method (see Labrecque et al. 2002, 2003; Svoboda et al. 2002). MMR measurements have also the potential to be made rapidly in free space, gaining a significant advantage over standard electric potential measurements collected in DC resistivity method (Yaramanci et al. 2005).

Another advantage of the MMR method is that when a conductivity anomaly is buried beneath a conductive layer, the DC resistivity method is not very sensitive to the conductivity contrast below the conductive layer while MMR exhibits less difficulty with this type of situation (see discussions in Edwards \& Howell 1976). MMR has also been used as a cross-hole imaging technique (Nabighian et al. 1984). Several applications using this method were developed in the realm of hydrogeophysics to detect solute plume transport mechanisms in the shallow subsurface (e.g. Kemna et al. 2002; Kulessa et al. 2002; Labrecque et al. 2002, 2003) and contamination of the 
ground water (e.g. Zhu \& Yang 2008). MMR has also been used in medical imaging (see for instance Levy et al. 2002).

In this paper, we use MMR in a very different way compared with the methods proposed above. The proposed and innovative approach developed below is closer to the classical idea of potential field methods. Acosta \& Worthington (1983) pointed out the sensitivity of MMR to current that is concentrated within a conductive anomaly. We inject the current between two electrodes A and B and our goal is to use MMR to visualize directly the current path in the ground in area of high conduction paths rather than to reconstruct the conductivity field per se. When the induction effects are neglected, we will show that the solution of this problem is represented by a vectorial Poisson equation, which is used to linearly relate the current density to the observed magnetic field.

This paper is organized as follows. We first discuss the background physics of MMR as well as the conditions under which a permeable path (the target) can be associated to a current flow. We also discuss how the current electrodes A and B should be strategically located to detect such flow path. Then we formulate the inverse problem. The sensitivity of the method is explored using five synthetic tests. Finally, we apply the MMR technology to identify preferential flow paths in the vicinity of a mine waste rock dump.

\section{THEORETICAL BACKGROUND OF MAGNETOMETRIC RESISTIVITY}

\subsection{Electrical conductivity}

The current flow paths in the ground are controlled by the electrical conductivity distribution and the position of the current electrodes A and B (Fig. 1). In the second part of this paper, we will discuss the MMR approach to current flow paths associated with preferential flow paths in the context of a mine waste rock dumps (Fig. 2). When surface conductivity is small (e.g. in presence of saline fluids), the conductivity of a water-saturated porous rock $\sigma$ (in $\mathrm{S} \mathrm{m}^{-1}$ ) is simply given by (e.g. Waxman \& Smits 1968):

$\sigma=\frac{1}{F} \sigma_{\mathrm{w}}+\sigma_{\mathrm{S}}$

where $F$ denotes the formation factor (dimensionless), $\sigma_{\mathrm{S}}$ denotes a quantity called surface conductivity and $\sigma_{\mathrm{w}}\left(\mathrm{S} \mathrm{m}^{-1}\right)$ denotes the pore water conductivity. In this case, the electrical conductivity is strongly controlled by the porosity of the material. A preferential flow path is expected to be associated with an area of high porosity and therefore high electrical conductivity and high permeability path. Indeed, the permeability $k$ (in $\mathrm{m}^{2}$ ) is related to the formation factor $F$ as:

$k=\frac{\Lambda^{2}}{8 F}$.

where $\Lambda$ (in $\mathrm{m}$ ) denotes a characteristic pore size of the material (Johnson et al. 1986).

Since our goal in hydrogeophysics is to use MMR to identify flow paths, it is important to know when a conductive path can be interpreted as a preferential flow path. Electrical conductivity and permeability are usually positively correlated when the first term of eq. (1) dominates while conductivity and permeability are anticorrelated when surface conductivity dominates the overall conductivity response (Purvance \& Andricevic 2000a,b). Therefore a conductive body in a given direction may be a preferential flow path if the head gradient is in the same direction and if the pore water conductivity

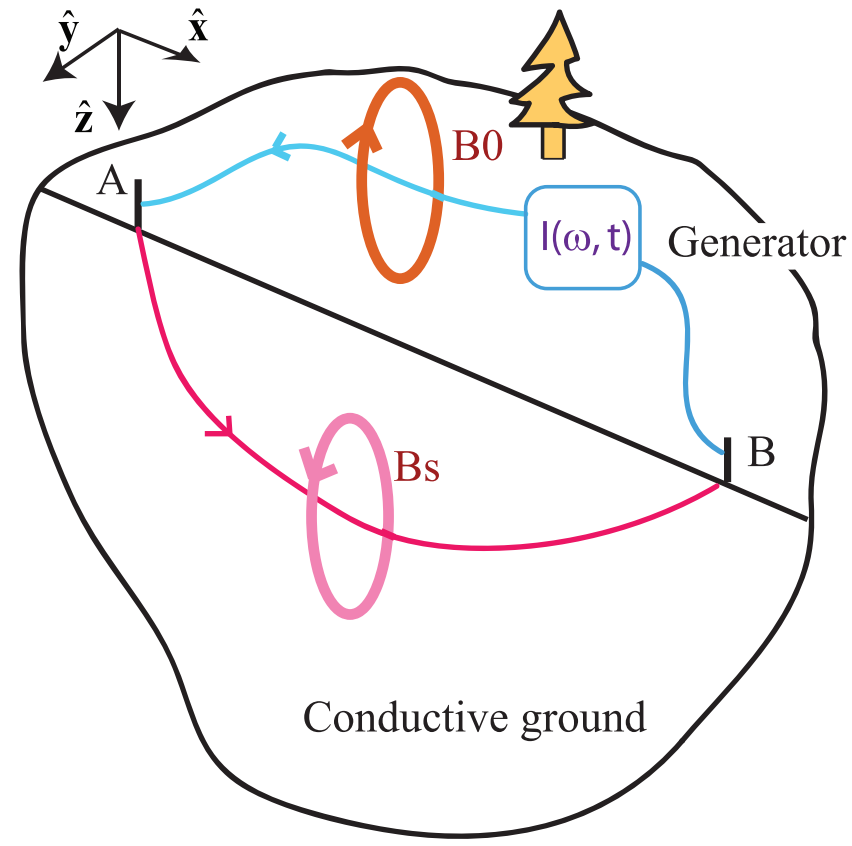

Figure 1. Sketch of the primary and secondary magnetic fields. A and B denote the two current electrodes (sometimes called the energizing electrodes in the terminology of Jakosky 1940). The generator produced an alternative current $I(\omega, t)$ where $\omega$ denotes the pulsation frequency. The field $\mathbf{B}_{0}$ is due to the cable lying along the ground surface and connected to the generator. In order to minimize its effect, this cable should be located as far as possible from the area of investigation. The field $\mathbf{B}_{0}$ is essentially vertical in the zone of interest between A and B. The field $\mathbf{B}_{\mathrm{S}}$ is the secondary field due to the current flowing in the ground, which can be or not localized depending on the electrical conductivity distribution. The measured magnetic field is the sum of these two magnetic fields. The area of investigation is comprised between the two energizing electrodes A and B. On a flat half conducting space, the primary field creates a magnetic field that is essentially vertical in the area of interest while the secondary magnetic field $\mathbf{B}_{\mathrm{S}}$ will have different components, mostly horizontal.

is rather high. This will be the case for the type of applications envisioned in this paper.

\subsection{Theory of MagnetoMetric resistivity}

The MMR method consists of injecting an electrical current between two electrodes, either at the surface or in boreholes, and measuring the magnetic field intensity along three orthogonal vectors. Thus, the forward problem of MMR resistivity involves combining the Maxwell field equations in the quasi-static conditions (i.e. time derivative is neglected in the conservation equations). In this situation, the Maxwell equations are simplified to (e.g. Chen et al. 2002):

$\nabla \times \mathbf{E}=0$,

$\nabla \cdot \mathbf{B}=0$

$\nabla \times \mathbf{B}=\mu \mathbf{J}_{c}$,

where $\mathbf{B}=\mu \mathbf{H}$ is the magnetic induction vector (in $\mathrm{T})$ ( $\mathbf{B}$ is more properly called the magnetic flux density), $\mathbf{H}$ is the magnetic field $\left(\mathrm{A} \mathrm{m}^{-1}\right), \mu$ is the magnetic permeability of free space $\left(4 \pi \times 10^{-7} \mathrm{H}\right.$ $\mathrm{m}^{-1}=4 \pi \times 10^{-7} \mathrm{~T} \mathrm{~m} \mathrm{~A}^{-1}$ ) and $\mathbf{J}_{c}$ denotes the conduction current density. 


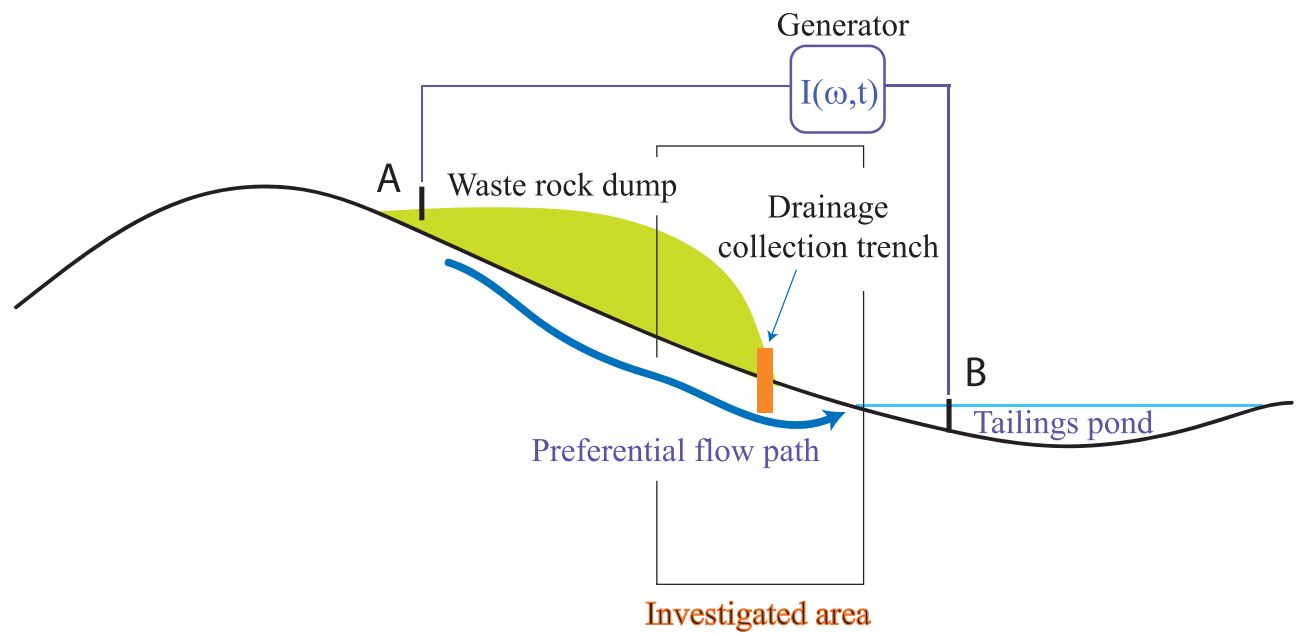

Figure 2. Sketck of the geometry of the energizing electrodes A and B to map a preferential ground water flow path passing a mine waste rock dump drainage collection trench to the tailings pond. The A and B current electrodes are located up-gradient and down-gradient of the drainage collection trench, respectively. An Alternative Current (AC) electrical current with a frequency of $380 \mathrm{~Hz}$ is applied to these two electrodes. As the electric current flow between A and B, it generates a magnetic field measured at the ground surface. The magnetic field is used to identify the location of preferential electric current flow paths. By identifying the electrically conductive flow paths (containing highly mineralized pore waters) between the strategically placed energizing electrodes A and B, preferential flow paths bypassing the drainage collection trench into the tailings pond can be identified. The investigated area corresponds to the zone where the three components of the magnetic fields are recorded at the ground surface of the Earth.

As a consequence of eq. (3), the electrical field can be derived from a scalar potential $\varphi$ (in $\mathrm{V}$ ) by $\mathbf{E}=-\nabla \varphi$. In a medium with current generators, the total current can be decomposed in two parts: a primary current flow related to the current generators $\mathbf{J}_{S}$ (source current density) and a volume current flow due to the electric field in the volume:

$\mathbf{J}_{c}=\sigma \mathbf{E}+\mathbf{J}_{S}=-\sigma \nabla \varphi+\mathbf{J}_{S}$,

where $\mathbf{J}_{S}$ represents the source current density (also called the primary current density) inside the source region and $\sigma \mathbf{E}$ is the current density outside the source region. The conservation equation for the charge is given by:

$\nabla \cdot \mathbf{J}_{c}=0$.

It follows that the electrical potential obeys the following elliptic partial differential equation:

$\nabla \cdot(\sigma \nabla \varphi)=\mathfrak{s}$,

where the volumetric source current density $\Im$ is represented as:

$\mathfrak{s}=\nabla \cdot \mathbf{J}_{S}$,

$\nabla \cdot \mathbf{J}_{S}=I\left[\delta\left(\mathbf{r}-\mathbf{r}_{A}^{+}\right)-\delta\left(\mathbf{r}-\mathbf{r}_{B}^{-}\right)\right]$,

where $\mathbf{r}$ denotes the position vector, $\mathbf{r}_{A}^{+}$and $\mathbf{r}_{B}^{-}$denotes the position of the current source (injection of the current in A) and sink (current retrieval in B), respectively. From eqs (8) to (10), the Poisson equation can be reformulated as:

$\nabla \cdot(\sigma \nabla \varphi)=I\left[\delta\left(\mathbf{r}-\mathbf{r}_{A}^{+}\right)-\delta\left(\mathbf{r}-\mathbf{r}_{B}^{-}\right)\right]$.

Eq. (11) can be solved numerically using finite element or finite difference methods with the following boundary conditions (Dey \& Morrison 1979):

$\hat{\mathbf{n}} \cdot(\sigma \cdot \nabla \varphi)=0$, at $\Gamma_{0}$,

$\varphi=0$, at $\Gamma_{i}$, where $\Gamma_{0}$ denotes the ground surface (characterized by an insulating boundary condition), $\hat{\mathbf{n}}$ is the unit normal vector to $\Gamma_{0}$, and $\Gamma_{i}$ denotes the other boundaries (infinite half-space boundaries).

We look now for a partial differential equation for the magnetic induction vector $\mathbf{B}$ and its integral form solution. Using Ampère's law, we have:

$\nabla \times \mathbf{B}=\mu \mathbf{J}_{c}=\mu\left(\mathbf{J}_{S}-\sigma \nabla \varphi\right)$,

and therefore, applying the curl to each side of eq. (14) and using eq. (13), we obtain:

$\nabla^{2} \mathbf{B}=-\mu \nabla \times \mathbf{J}_{c}$,

$\nabla^{2} \mathbf{B}=-\mu \nabla \times\left(\mathbf{J}_{S}-\sigma \nabla \varphi\right)$.

Note that $\nabla \cdot(\nabla \times \mathbf{B})=\nabla \cdot\left(\mu \mathbf{J}_{c}\right)=\mu \nabla \cdot \mathbf{J}_{c}=0 \quad$ since $\nabla \cdot \mathbf{J}_{c}=0$. Because $\nabla \cdot \mathbf{B}=0$, there exists a vector field $\mathbf{A}$ such that: $\mathbf{B}=\nabla \times \mathbf{A}$ (A denotes the magnetic vector potential). We use the classical gauge condition $\nabla \cdot \mathbf{A}=0$ to avoid the indetermination caused by the definition of $\mathbf{A}$. Maxwell's equation $\nabla \times \mathbf{B}=\mu \mathbf{J}_{c}$ becomes $\Delta \mathbf{A}=\mu \mathbf{J}_{c}$, which is a vectorial Poisson equation. If we impose $\mathrm{A}(|\mathrm{r}| \rightarrow \infty)=0$ (no magnetic induction $\mathbf{B}$ at infinity), it has a general solution at the observation point $\mathrm{P}(\mathbf{r})$ and in 3-D:

$$
\mathbf{A}(\mathbf{r})=\frac{\mu}{4 \pi} \int_{\Omega} \frac{\mathbf{J}_{c}\left(\mathbf{r}^{\prime}\right)}{\left|\mathbf{r}-\mathbf{r}^{\prime}\right|} \mathrm{d} \tau^{\prime},
$$

where $\mathbf{r}$ denotes the position of the observation point $\mathrm{P}, \mathrm{r}^{\prime}$ denotes the position of the integration point around the source point $M$, and $\mathrm{d} \tau^{\prime}$ denotes a volume element around the source point $\mathrm{M}$. For $\mathrm{MMR}$, it can be interesting to compute $\mathbf{A}$ from the current density (since the corresponding Green function is very simple) and then to compute the curl of $\mathbf{A}$ to get the vector field $\mathbf{B}$. Taking the curl, we obtain the Biot and Savart law (e.g. Nabighian 1991a,b):

$$
\begin{aligned}
\mathbf{B}(\mathbf{r}) & =\frac{\mu}{4 \pi} \int \frac{\mathbf{J}_{c}\left(\mathbf{r}^{\prime}\right) \times\left(\mathbf{r}-\mathbf{r}^{\prime}\right)}{\left|\mathbf{r}-\mathbf{r}^{\prime}\right|^{3}} \mathrm{~d} \tau^{\prime} \\
& =\frac{\mu}{4 \pi} \int \mathbf{J}_{c}\left(\mathbf{r}^{\prime}\right) \times \nabla^{\prime}\left[\frac{1}{\left|\mathbf{r}-\mathbf{r}^{\prime}\right|}\right] \mathrm{d} \tau^{\prime},
\end{aligned}
$$




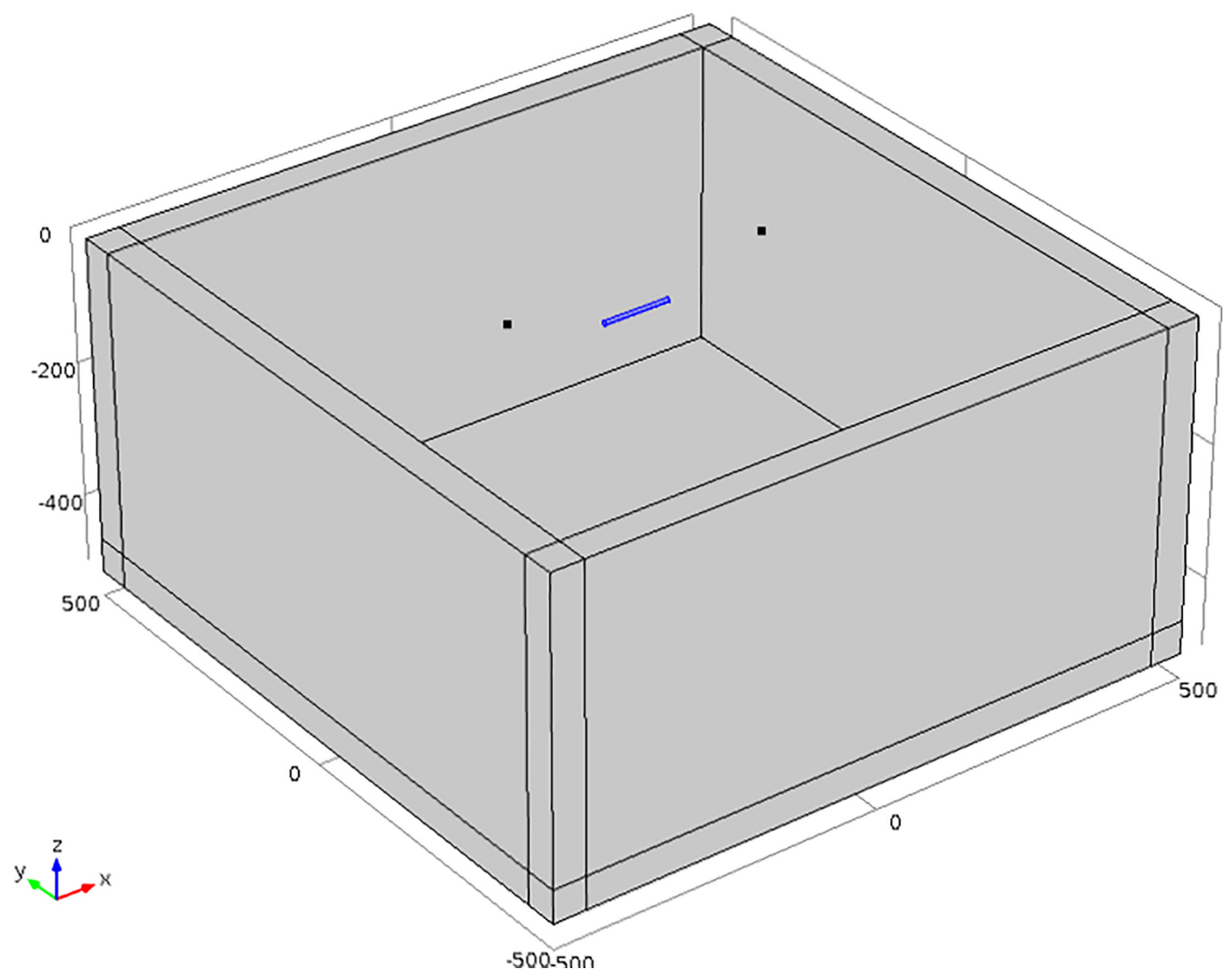

Figure 3. Geometry of the investigated domain used to study the sensitivity of the MMR method to identify the heterogeneity of the electrical resistivity of subsurface, here presented by a conductive anomaly buried in a homogeneous medium with an electrical resistivity of $1000 \mathrm{Ohm} \mathrm{m}$. The values of the resistivity of the anomaly, its depth and its radius are changed to see their impacts on the magnetic field signature. We mention that the electrodes A and B used to inject electrical current are placed at the ground surface and materialized in the figure by the two points.

$\mathbf{B}(\mathbf{r})=\frac{\mu}{4 \pi} \int\left(\mathbf{J}_{S}\left(\mathbf{r}^{\prime}\right)-\sigma\left(\mathbf{r}^{\prime}\right) \nabla \varphi\left(\mathbf{r}^{\prime}\right)\right) \times \frac{\mathbf{r}-\mathbf{r}^{\prime}}{\left|\mathbf{r}-\mathbf{r}^{\prime}\right|^{3}} \mathrm{~d} \tau^{\prime}$.

At the observation point $\mathrm{P}(\mathbf{r})$ (position of the magnetometer), the magnetic field can be decomposed as (e.g. Chen et al. 2002):

$\mathbf{B}(\mathbf{r})=\mathbf{B}_{0}(\mathbf{r})+\mathbf{B}_{S}(\mathbf{r})$

$\mathbf{B}_{0}(\mathbf{r})=\frac{\mu}{4 \pi} \int \mathbf{J}_{S}\left(\mathbf{r}^{\prime}\right) \times \frac{\mathbf{r}-\mathbf{r}^{\prime}}{\left|\mathbf{r}-\mathbf{r}^{\prime}\right|^{3}} \mathrm{~d} \tau^{\prime}$,

$\mathbf{B}_{S}(\mathbf{r})=\frac{\mu}{4 \pi} \int\left(\sigma\left(\mathbf{r}^{\prime}\right) \mathbf{E}\left(\mathbf{r}^{\prime}\right)\right) \times \frac{\mathbf{r}-\mathbf{r}^{\prime}}{\left|\mathbf{r}-\mathbf{r}^{\prime}\right|^{3}} \mathrm{~d} \tau^{\prime}$.

There is also another magnetic field due to the wire lying aong the ground surface and used to connect the current electrodes $\mathrm{A}$ and $\mathrm{B}$ to the generator. We will discuss how to remove this spurious contribution below. With this formulation, $\mathbf{B}_{0}$ is called the primary magnetic field (normal magnetic field due to current injection in the soil) while the second term $\mathbf{B}_{S}(\mathbf{r})$ is called the secondary magnetic field. The field $\mathbf{B}_{0}$ depends only on the amplitude and positions of the electrical courant sources A and B.

In MMR, the source of current controlling $\mathbf{J}_{S}$ is known and is imposed, for example, through the Willowstick technology using two electrodes, a source current of 1 to $2 \mathrm{~A}$ with an $\mathrm{AC}$ frequency of $380 \mathrm{~Hz}$. Therefore, we can easily remove the primary field and look at the residual (secondary) field $\mathbf{B}_{S}(\mathbf{r})=\mathbf{B}(\mathbf{r})-\mathbf{B}_{0}(\mathbf{r})$ :

$\mathbf{B}_{S}(\mathbf{r})=\frac{\mu}{4 \pi} \int(\sigma \mathbf{E}) \times \frac{\mathbf{r}-\mathbf{r}^{\prime}}{\left|\mathbf{r}-\mathbf{r}^{\prime}\right|^{3}} \mathrm{~d} \tau^{\prime}$.
Developing this equation using $\nabla \times \mathbf{E}=0$ everywhere (in the quasi-static limit discussed above), we have:

$\mathbf{B}_{S}(\mathbf{r})=-\frac{\mu}{4 \pi} \int_{\Omega} \frac{\nabla^{\prime} \sigma\left(\mathbf{r}^{\prime}\right) \times \mathbf{E}\left(\mathbf{r}^{\prime}\right)}{\left|\mathbf{r}-\mathbf{r}^{\prime}\right|} \mathrm{d} \tau^{\prime}$.

$\mathbf{B}_{S}(\mathbf{r})=\frac{\mu}{4 \pi} \int_{\Omega} \frac{\nabla^{\prime} \sigma\left(\mathbf{r}^{\prime}\right) \times \nabla \varphi\left(\mathbf{r}^{\prime}\right)}{\left|\mathbf{r}-\mathbf{r}^{\prime}\right|} \mathrm{d} \tau^{\prime}$.

For a homogeneous subsurface $\left(\nabla^{\prime} \sigma\left(\mathbf{r}^{\prime}\right)=0\right)$, the magnetic field reduces to the primary field $\mathbf{B}_{0}$ (the secondary magnetic field is null). From eq. (25), we see that the MMR is sensitive to the gradient of the electrical conductivity distribution. If the flow path is characterized by a strong drop in the electrical conductivity with the surrounding homogeneous material, it will be detectable through the magnetic measurements.

\section{FORWARD AND INVERSE PROBLEMS}

\subsection{Forward problem}

In this section, we formulate the forward problem by using the discretization form of Biot and Savart's law, see eq. (23) and where we apply $\mathbf{J}_{c}$ only to the subsurface to get the secondary magnetic field

$\mathbf{B}_{S}(\mathbf{r})=\frac{\mu}{4 \pi} \int \frac{\mathbf{J}_{c}\left(\mathbf{r}^{\prime}\right) \times\left(\mathbf{r}-\mathbf{r}^{\prime}\right)}{\left|\mathbf{r}-\mathbf{r}^{\prime}\right|^{3}} \mathrm{~d} \tau^{\prime}$, 

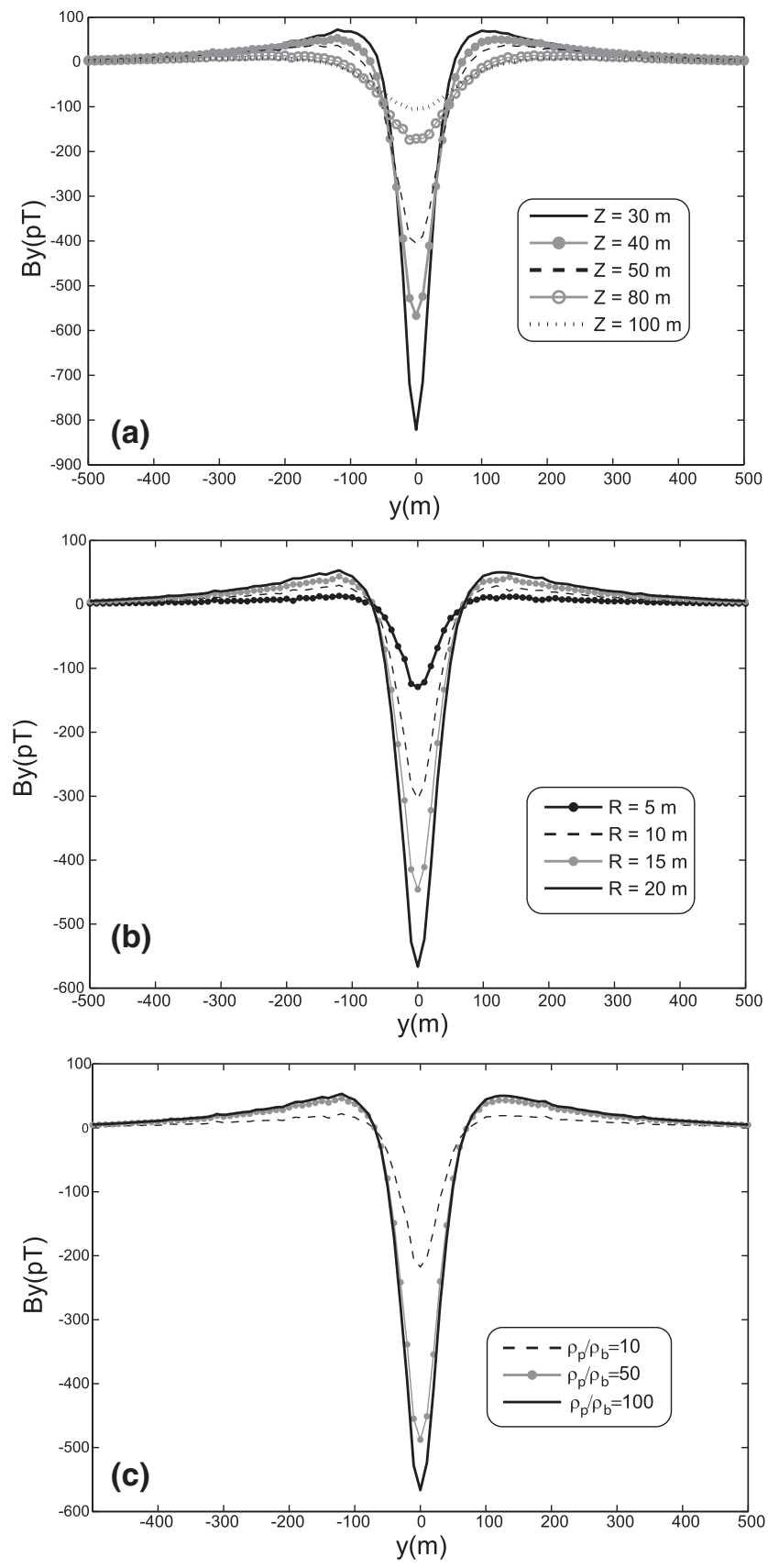

Figure 4. Signature of horizontal ( $y$-) component of the secondary magnetic field (in pT) induced by a contrast of the electrical resistivity between the background and the anomaly. This signature is extracted from the profile crossing the center of the domain $x=0 \mathrm{~m}$. (a) Effect of the depth of heterogeneous anomaly on the amplitude of the magnetic signal, which decreases with the increase of the depth of the target. (b). The amplitude of the magnetic signal increases with the increase in the volume of the anomaly. (c). Behavior of the magnetic signal with the contrast of resistivity between the anomaly and background. The amplitude of the magnetic field increases with the contrast of resistivity.

where $\mathbf{r}-\mathbf{r}^{\prime}$ define the separation vector between the source point $M\left(\mathbf{r}^{\prime}\right)$ and the observation point $P(\mathbf{r})$. We can proceed to discretization. We assume the subsurface is discretized with $M$ small cells having all the same volume $v$ and constant current density in each cell. For a single cell $\mathrm{j}$ and a single observation point $\mathrm{i}$, the magnitude of the magnetic field can be expressed as:

$$
\begin{aligned}
& {\left[\begin{array}{c}
B_{x}^{S, i} \\
B_{y}^{S, i} \\
B_{z}^{S, i}
\end{array}\right]=\frac{\mu v}{4 \pi r_{i j}{ }^{3}}\left[\begin{array}{c}
J_{y}^{j} r_{z}^{i j}-J_{z}^{j} r_{y}^{i j} \\
J_{z}^{j} r_{x}^{i j}-J_{x}^{j} r_{z}^{i j} \\
J_{x}^{j} r_{y}^{i j}-J_{y}^{j} r_{x}^{i j}
\end{array}\right],} \\
& {\left[\begin{array}{c}
B_{x}^{S, i} \\
B_{y}^{S, i} \\
B_{z}^{S, i}
\end{array}\right]=\frac{\mu v}{4 \pi r_{i j}{ }^{3}}\left[\begin{array}{ccc}
0 & r_{z}^{i j} & -r_{y}^{i j} \\
-r_{z}^{i j} & 0 & r_{x}^{i j} \\
r_{y}^{i j} & -r_{x}^{i j} & 0
\end{array}\right]\left[\begin{array}{c}
J_{x}^{j} \\
J_{y}^{j} \\
J_{z}^{j}
\end{array}\right],}
\end{aligned}
$$

where $r_{i j}$ is the distance between the cell $\mathrm{j}$ to the observation point $\mathrm{i}$ where the magnetic measurement is performed. We assume now that we have $3 \mathrm{~N}$ observation points including the three components of the magnetic fields $\mathbf{B}_{s}$ and $M$ cells used to discretize the vector of electrical current density in subsurface $\mathbf{J}$, we have therefore $\mathbf{B}_{s}=\mathbf{K J}$, where $\mathbf{K}$ is the kernel matrix $(3 N \times 3 M)$ describing a linear relationship between the observed magnetic field and the vector of source current density. $\mathbf{K}$ is typically computed by putting elementary current sources in each cell of the discretized subsurface and along each of the three principal directions of the coordinate system and computing the resulting magnetic field at the position of the recording stations located at the ground surface. Starting with the discretized form of eq. (26), we can express the predicted data from a source current distribution as:

$\mathbf{d}_{p}=\mathbf{K} \mathbf{m}$,

where $\mathbf{d}_{p}$ denote the $3 N$-vector of predicted magnetic field and $\mathbf{m}$ is the $3 M$-vector of unknown source current density. The problem is linear and we can use all the approaches developed in potential field tomography to solve this problem (see Jardani et al. 2008 for the self-potential problem and Pedersen 1977, for the gravity field problem).

\subsection{Inverse problem}

The two forms given in the previous section can be written in discretized linear form as $\mathbf{d}_{p}=\mathbf{K} \mathbf{m}$. After establishing the kernel, the objective function $P^{\lambda}(\mathbf{m})$ can be defined as the sum of two terms, a data misfit function and a regulation function, that is

$P^{\lambda}(\mathbf{m})=\left\|\mathbf{W}_{d}\left(\mathbf{K m}-\mathbf{d}_{o}\right)\right\|^{2}+\lambda\left\|\mathbf{W}_{m}\left(\mathbf{m}-\mathbf{m}_{0}\right)\right\|^{2}$,

both balanced by a regularization parameter $\lambda$. In eq. (30), $\mathbf{m}$ denotes the $3 M$-vector model characterizing the source current density distribution in the ground, $\mathbf{d}_{o}$ denotes the observed data $3 N$-vector of the secondary magnetic field, $M$ and $N$ describe the number of cells of the model and the number of stations where the three components of the magnetic field are measured (typically $M>>3 N$ and the problem is generally underdetermined), $\mathbf{m}_{0}$ is a prior reference $3 \mathrm{M}$ model vector (when we have no prior information this vector is set up to the null vector), $\mathbf{W}_{\mathrm{m}}$ denotes the regularization matrix used to assure a stable result, and $\mathbf{W}_{d}$ denotes the inverse of the data covariance matrix. There are various ways to generate $\mathbf{m}_{0}$. For instance, $\mathbf{m}_{0}$ can be built with a 3-D electrical conductivity model (using ERT, electrical resistance tomography) and simulating the current injected between A and B. 
(a)

\section{Case study 1}

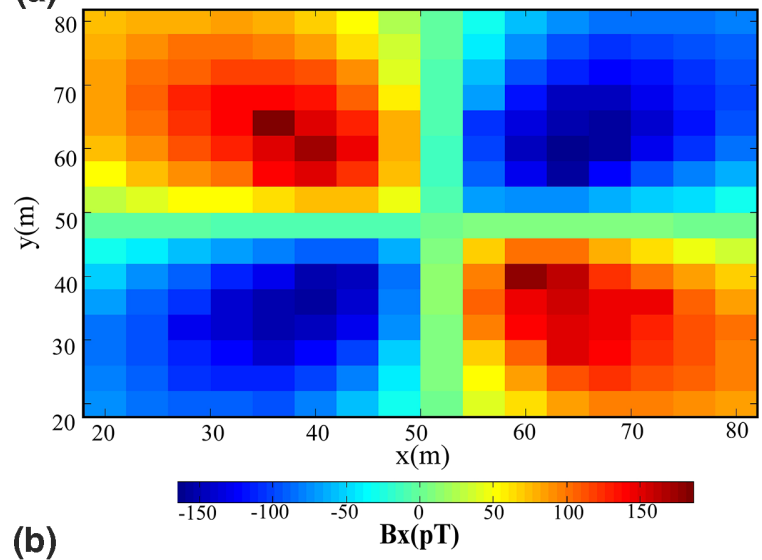

(b)

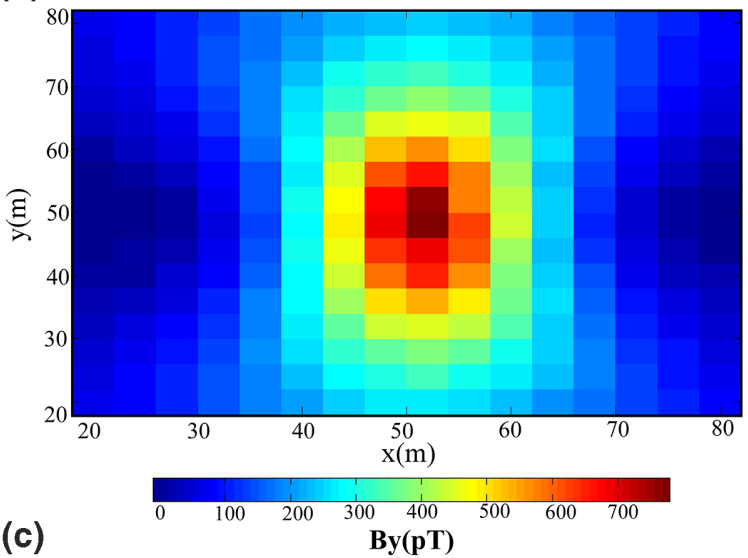

(c)

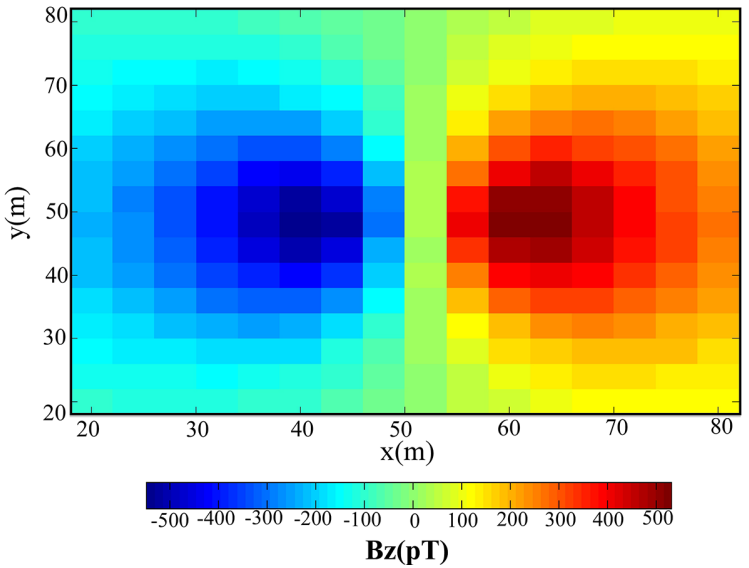

(d)

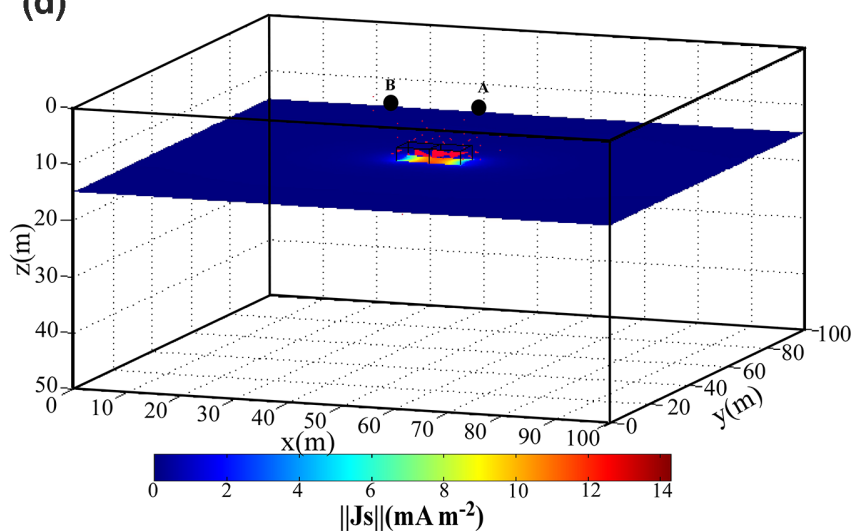

(e)

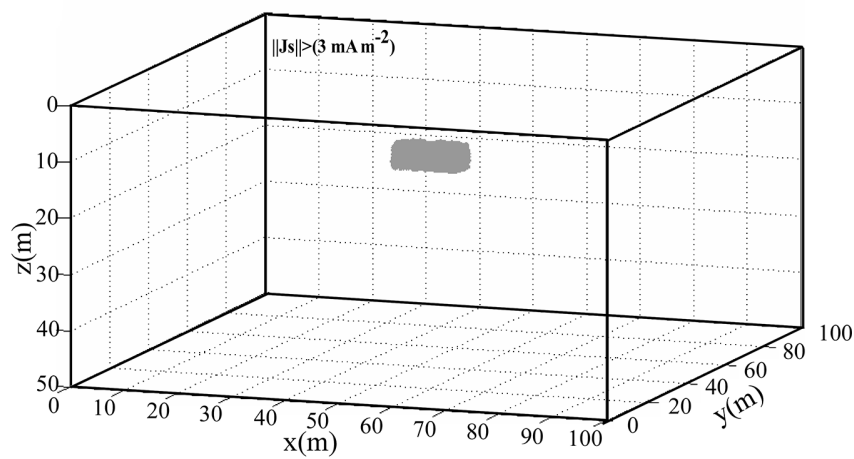

(f)

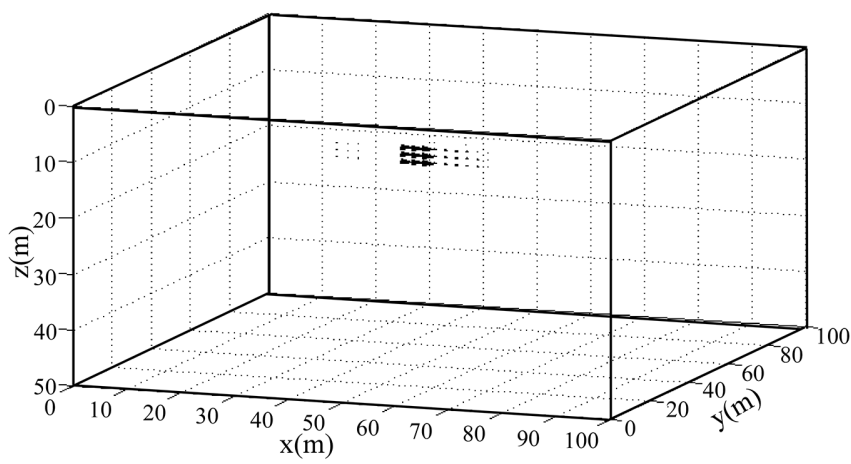

Figure 5. Simulation of the first case study, $(\mathrm{a}-\mathrm{c})$ Distribution of the three components of secondary magnetic field generated by the presence of the conductive block $(10 \mathrm{Ohm} \mathrm{m})$ with a dimension of $12 \mathrm{~m} \times 6 \mathrm{~m} \times 4 \mathrm{~m}$ in a resistive background $(1000 \mathrm{Ohm} \mathrm{m})$, (d) The primary field is created by the injection of an electric current of 2A between two electrodes A and B separated by a distance of $40 \mathrm{~m}$. This heterogeneity of electrical resistivity produces a secondary current density for which the horizontal component $J_{x}$ is the main source behind the magnetic signature. (e) The inversion of the magnetic data permits the reconstruction of the secondary current density, thus the shape and position of the heterogeneity as shown by the plot of electrical current density norm presented in Fig.4(e). (f) The dominant direction of the electrical current density $J x$ is reproduced in this inversion result.

The solution of the previous linear inverse problem is wellestablished and is given by (e.g. Hansen 1998):

$$
\begin{aligned}
\mathbf{m}= & {\left[\mathbf{K}^{T}\left(\mathbf{W}_{d}^{T} \mathbf{W}_{d}\right) \mathbf{K}+\lambda\left(\mathbf{W}_{m}^{T} \mathbf{W}_{m}\right)\right]^{-1} } \\
& \times\left[\mathbf{K}^{T}\left(\mathbf{W}_{d}^{T} \mathbf{W}_{d}\right) \mathbf{d}_{o}+\lambda\left(\mathbf{W}_{m}^{T} \mathbf{W}_{m}\right) \mathbf{m}_{0}\right] .
\end{aligned}
$$

Depth weighting of the kernel is needed to counterbalance the decay of the magnetic signal with the increase of the depth, and a diagonal weighting matrix can be defined as (Li \& Oldenburg 1996, 1998,
2000):

$\boldsymbol{\Lambda}=\operatorname{diag}\left[\left(z+z_{0}\right)^{-\gamma / 2}\right]$,

where 'diag' means creating a diagonal matrix $(3 M \times 3 M), z$ is depth of the center of $M$ cells used to assign the electrical current density sources, $z_{0}$ is the altitude of the observation points, and $\gamma$ is a parameter that enables reproduction of the decreasing behavior of the kernel matrix. 
Table 1. Parameters used in the inversion of the five synthetic case studies and quality of the fitting of observed data based on the norm of the difference between predicted and observed data. The resistivity contrast denotes the ratio between the resistivity of the target and the resistivity of the background.

\begin{tabular}{lccccc}
\hline & Case 1 & Case 2 & Case 3 & Case 4 & Case 5 \\
\hline Partitioning & $6 \times 6 \times 4$ & $6 \times 6 \times 4$ & $6 \times 6 \times 4$ & $6 \times 6 \times 4$ & $4 \times 4 \times 4$ \\
Resistivity contrast & $10 / 1000$ & $100 / 1000$ & $10 / 1000$ & $10 / 1000$ & $10 / 1000$ \\
$\mathbf{W}_{d}=\omega_{d} \mathbf{I}$ & $\omega_{d}=10^{3}$ & $\omega_{d}=10^{3}$ & $\omega_{d}=10^{7}$ & $\omega_{d}=10^{7}$ & $\left(10^{7}\right.$ \\
$(\lambda, \beta, \gamma)$ & $\left(10^{-10}, 10^{-6}, 1\right)$ & $\left(10^{-10}, 10^{-6}, 1\right)$ & $\left(10^{-4}, 10^{-6}, 3\right)$ & $\left(10^{-5}, 10^{-6}, 1\right)$ & $\left(10^{-5}, 10^{-8}, 0.6\right)$ \\
$\left\|\mathbf{W}_{d}\left(\mathbf{K m}-\mathbf{d}_{\text {obs }}\right)\right\|^{2}$ & $10^{-8}$ & $5 \times 10^{-10}$ & $1 \times 10^{-4}$ & $4 \times 10^{-5}$ & $1 \times 10^{-6}$ \\
\hline
\end{tabular}

The quantities $\lambda, \beta$ and $\gamma$ denote the regularization parameter, the parameter used to set up the source compaction, and depth weighting parameter, respectively.

The matrix $\mathbf{W}_{d}$ denotes the data covariance matrix and $\mathbf{I}$ the identity matrix.

The previous inversion scheme provides a smooth density distribution obtained from a linear current density inversion with the L2 norm. However, if the current density anomaly is expected to be rather localized, further compaction is required during the inversion process. Various strategies have been established for this purpose. We use the MS (minimum support) method shown by Last \& Kubik (1983). The MS functions are defined as:

$M S=\sum_{k=1}^{M} \frac{m_{k}^{2}}{m_{k(i-1)}^{2}+\beta^{2}}$,

where $m_{k(i-1)}$ is source current density at iteration $(i-1)$ and position $k$ and $\beta$ denotes a small threshold number in the minimum support method (see Zhdanov \& Tolstaya 2004).

With the MS function, a new diagonal weighting matrix can be established:

$\boldsymbol{\Omega}=\operatorname{diag}\left[\sqrt{\frac{\Lambda_{k k}^{2}}{m_{k-1}^{2}+\beta^{2}}}\right]$,

in which $\boldsymbol{\Omega}$ is the newly updated weighting matrix, $\Lambda_{k k}^{2}$ can be determined through eq. (32) The kernel matrix is revised as $\mathbf{K}^{*}=$ $\mathbf{W}_{d} \mathbf{K} \Omega^{-1}$, and eq. (31) is therefore revised to have a new form with $\mathbf{W}_{\mathrm{m}}=\mathbf{I}_{\mathrm{m}}(3 M$ identity matrix $)$ :

$\mathbf{m}^{*}=\left[\mathbf{K}^{*} T \mathbf{K}^{*}+\lambda \mathbf{I}_{m}\right]^{-1} \cdot\left[\mathbf{K}^{*} \mathbf{d}_{o}^{*}\right]$,

with $\mathbf{d}_{o}^{*}=\mathbf{W}_{d} \mathbf{d}_{o}$. This formulation can be expressed by using the singular value decomposition of the Kernel matrix as following (Hansen 1998):

$\mathbf{m}^{*}=\mathbf{V} \operatorname{diag}\left[\frac{s_{i}^{2}}{s_{i}^{2}+\lambda} \frac{1}{s_{i}}\right] \cdot\left[\mathbf{U}^{T} \mathbf{d}_{o}^{*}\right]$,

where $\mathbf{K}^{*}=\mathbf{U S} \mathbf{S}^{T}$ and $\mathbf{S}=\operatorname{diag}\left(s_{i}\right)$ where diag denotes a diagonal matrix of the argument. The retrieved source current density must to be transformed back after each iteration to get an unscaled current density according to $\mathbf{m}=\boldsymbol{\Omega}^{-1} \mathbf{m}^{*}$. The compaction process can be performed iteratively until the difference between two consecutive iterations is smaller than a prescribed value. A trade-off curve method is used to select $\beta$ by plotting the value of the logarithm of the data misfit versus the logarithm of the value of the regularizer for the current model estimate over a range of values for $\beta$. Then, we select the optimal value at the point of maximum curvature on such $\log -\log$ plot.

\section{SENSITIVITY ANALYSIS}

\subsection{Geometry}

The goal of this section is to study the sensitivity of the MMR as it pertains to detecting the contrast resistivity in different conditions.
We place in a large domain $(1000 \mathrm{~m} \times 1000 \mathrm{~m} \times 500 \mathrm{~m})$ with a uniform electrical resistivity $(1000 \mathrm{Ohm} \mathrm{m})$, a conductive path whose geometric parameters, position, and electrical resistivity values will be changed to see their impact on the signature of the secondary magnetic field calculated at the surface (Fig. 1). The model domain and conductive path (or 'pipe') is shown in Fig. 3. In Fig. 4(a), we illustrate the effect of the depth of pipe on the magnetic field by modifying the depth of the anomaly and fixing the contrast between the electrical resistivity of the anomaly $\rho_{p}$ and the conductivity of the background $\rho_{b}$ at $\rho_{p} / \rho_{b}=100$, and the radius of the anomaly at $R=20 \mathrm{~m}$.

These simulations show that the magnitude of the magnetic field decreases with increased depth. This decrease proportionally follows the tendency $\left(1 / r^{2}\right.$ where $r$ is the distance separating the subsurface magnetic sources and the observation position at the ground surface). The tests conducted on the impact of the pipe's geometry (radius) on the magnetic signal illustrate that amplitude of the signal increases with the radius (Fig. 4b). This is due to the increase in the volume of heterogeneity which favors the arising of an important secondary current density crossing this volume. The effect of the contrast in resistivity between the anomaly and background is presented in the Fig. 4(c) in which the amplitude of the magnetic field increases with the contrast of resistivity. These simple tests demonstrate that the effectiveness of MMR in the detection of conductive bodies will depend on the depth of these bodies, their geometry, and on the contrasts of resistivity with respect to the surrounding environment, especially the background conductivity.

\subsection{Case study 1}

We first test the efficiency of our inversion algorithm on a case study to locate a conductive heterogeneity by using the MMR data. We first place a conductive block $(6 \mathrm{~m} \times 6 \mathrm{~m} \times 4 \mathrm{~m})$ with electrical resistivity of $10 \mathrm{Ohm} \mathrm{m}$ at the center of a uniform domain $(50 \mathrm{~m} \times 50 \mathrm{~m} \times 14.5 \mathrm{~m})$ with an electrical resistivity of $1000 \mathrm{Ohm}$ $\mathrm{m}$ (Fig. 5). Its depth is $z=25 \mathrm{~m}$. The primary electrical current density is generated from two point-sources settled at $5 \mathrm{~m}$ from the ground surface. The anomalies of the magnetic field caused by the secondary electrical current density related to the heterogeneity (the conductive block) were recorded at the surface at $N=256$ locations at which we captured the three components of the magnetic field (Fig. 5). So the total number of observations is $3 N=768$. Then, this synthetic magnetic data is treated in the regularization inverse algorithm to determine the electrical current source by using the depth weight and compact constraints. The three components of electrical current sources are assigned to the cells that were used originally to partition the domain. Because the number of these cells is $M=3072$, then the number of unknown parameters to estimate is $3 \times 3072$; therefore we deal with a strongly underdetermined inverse problem. 


\section{Case study 2}

(a)
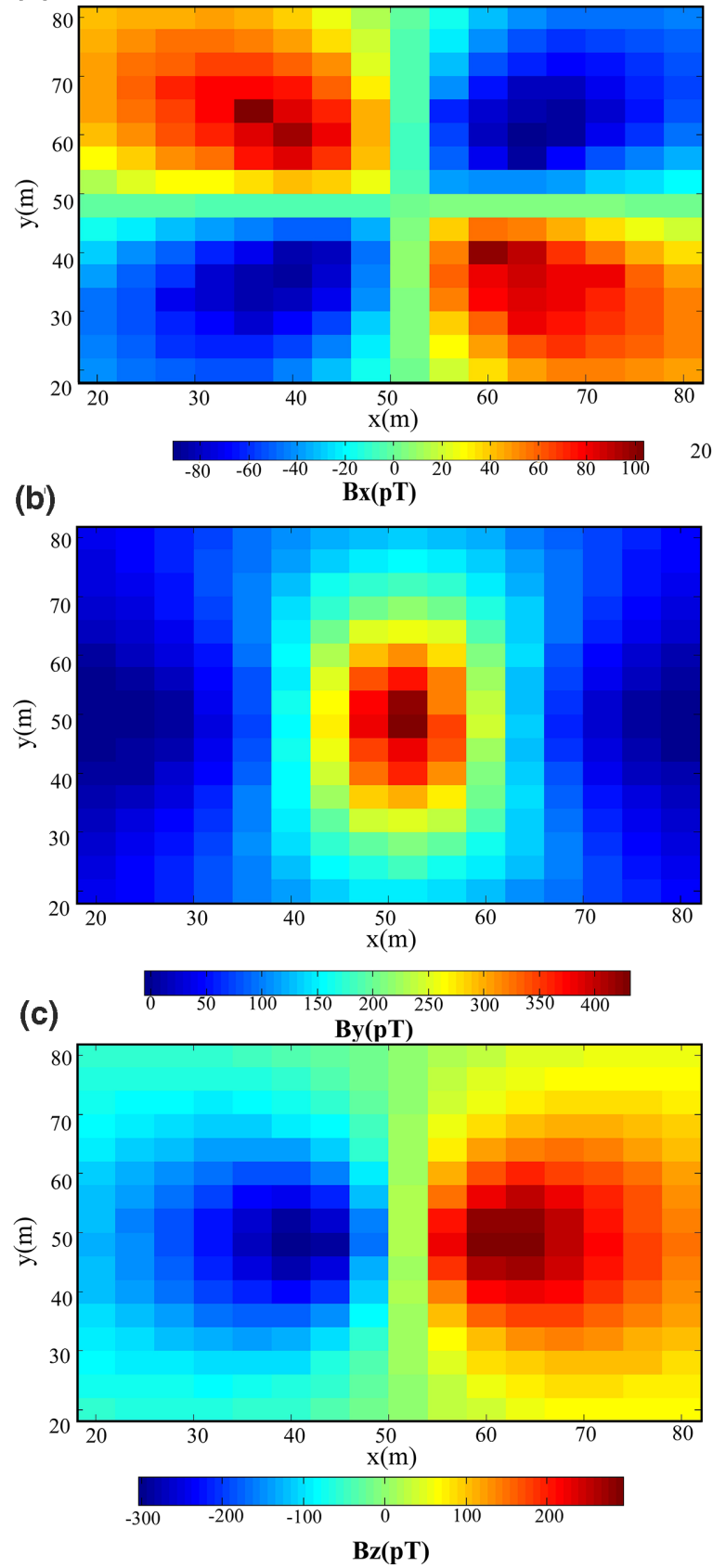

(d)
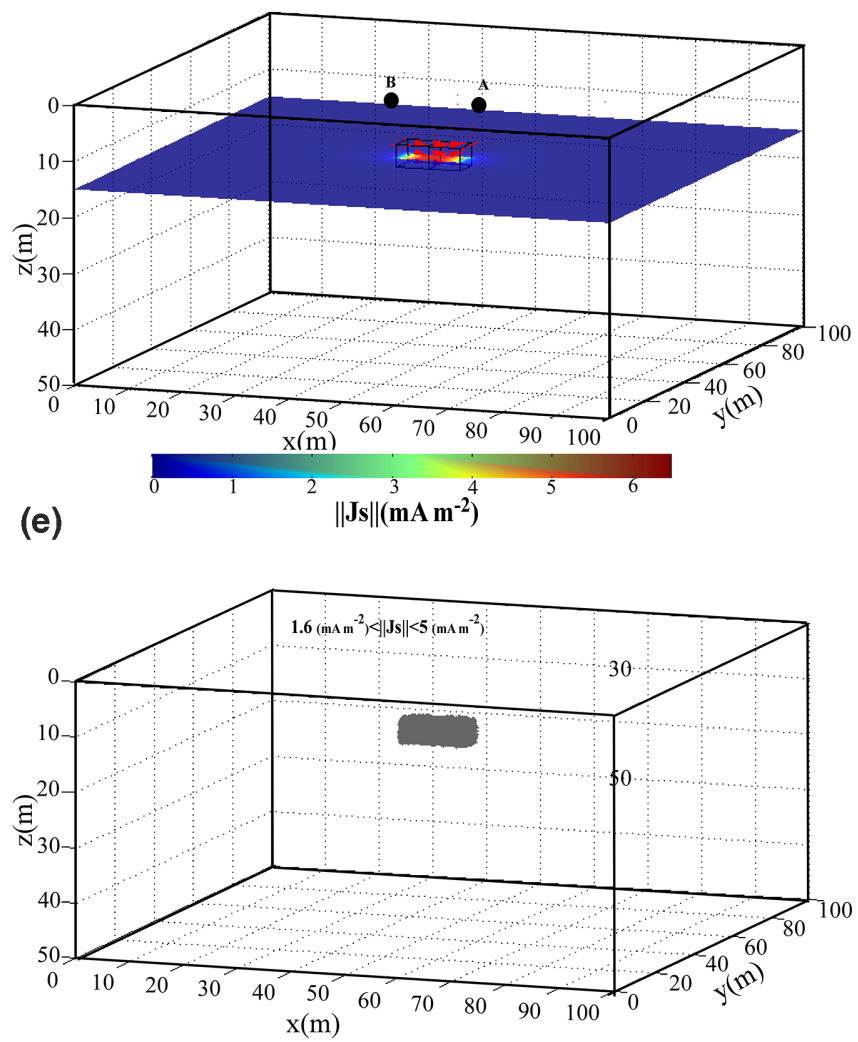

(f)

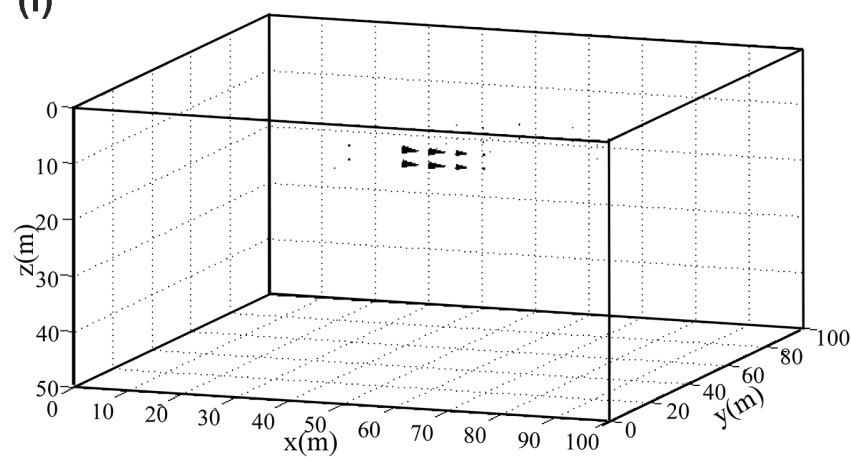

Figure 6. Simulation for the second case study with a depth of the anomaly of $25 \mathrm{~m}$. (a-c) Distribution of the three components $\left(B_{x}, B_{y}, B_{z}\right)$ of the secondary magnetic field due to a relatively less conductive block $(100 \mathrm{Ohm} \mathrm{m}$ ) with a dimension of $12 \times 6 \times 4 \mathrm{~m}$ placed in a resistive background (1000 Ohm $\mathrm{m})$. (d) The geometry of the block and the position of the electrodes used for current injection. (e) Result of the inversion process in which the norm of the electrical current density is reconstructed indirectly from the magnetic field data, which delineates the form of the heterogeneity despite the weakness of the electrical resistivity contrast. (f) The inversion identifies the main component of the current density $J x$ responsible for the magnetic signature.

The regularizer and compact parameters $\lambda$ and $\beta$ used to constraint the optimization were chosen in such a way as to establish a balance in the inversion between the data and the constraints. The procedure is based on the analysis of several results of the inversion with different values in the range $10^{-10} \leq \lambda \leq 10^{2}$ and $10^{-10} \leq \beta \leq 10^{-3}$. We recall that the high values of $\lambda$ promotes the optimization of regularization term $\left\|\mathbf{W}_{m}\left(\mathbf{m}-\mathbf{m}_{0}\right)\right\|^{2}$, that is the model obtained is more impacted by the a priori information than the observed data. The compaction parameter $\beta$ controls the spatial shape of the electrical current density, when the value of $\beta$ increases the shape of the inverted model becomes very compact. Thus both parameters are linked and we therefore optimized for a joint analysis to select the best parameters. The various parameters used in the inverse problem (for all five cases) are reported in Table 1. 
(a)

\section{Case study 3}
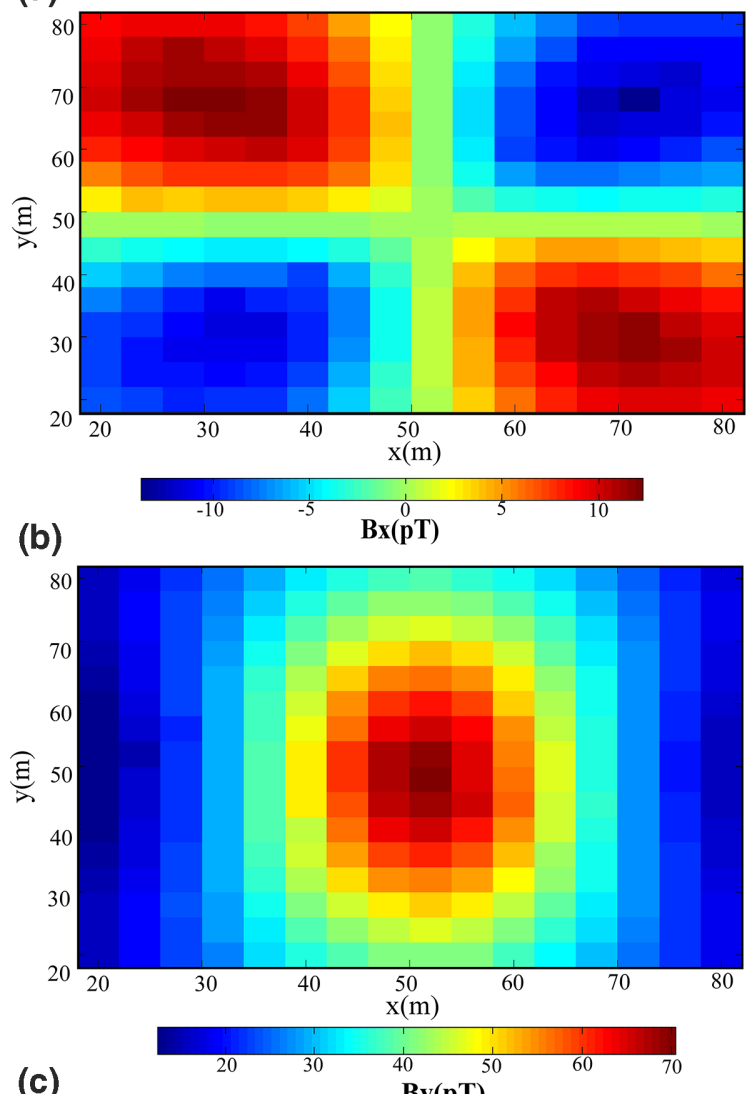

(c)

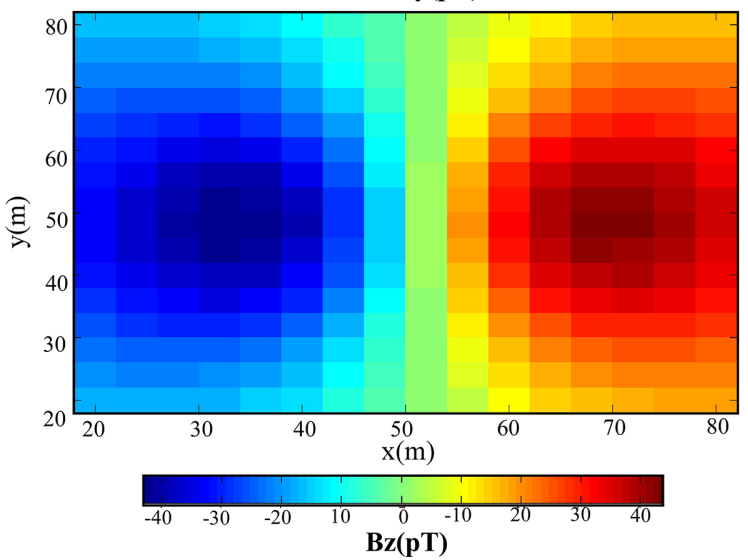

(d)
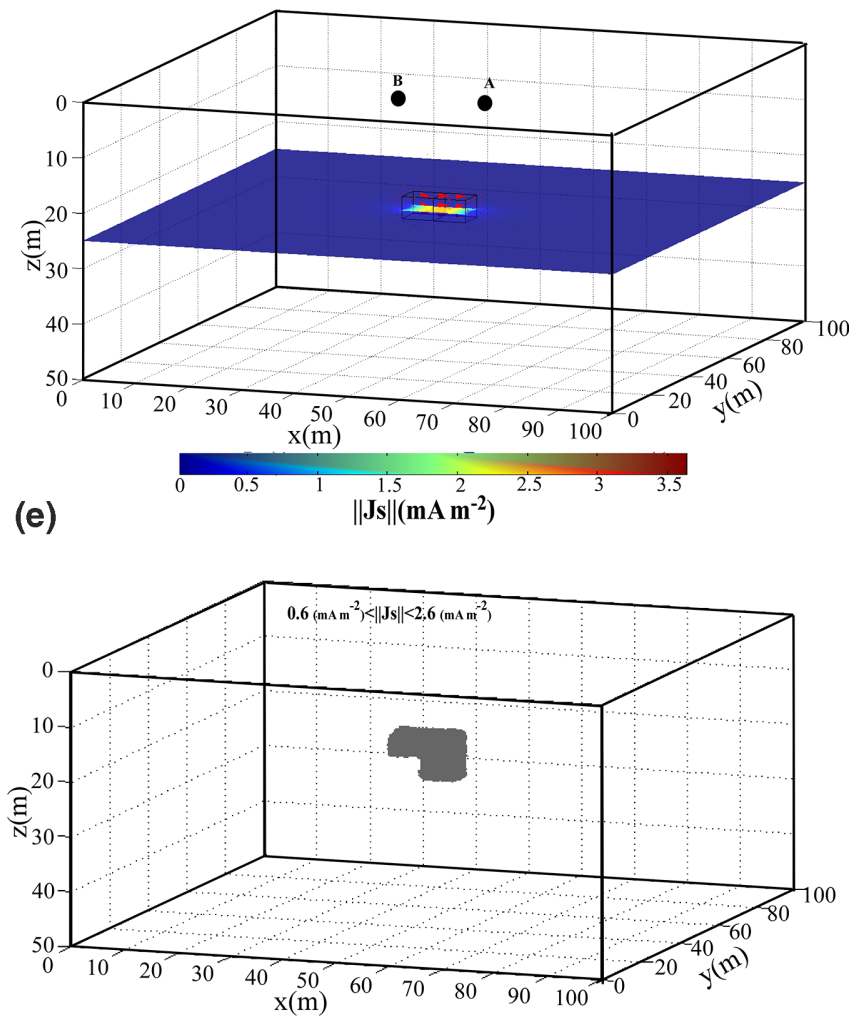

(f)

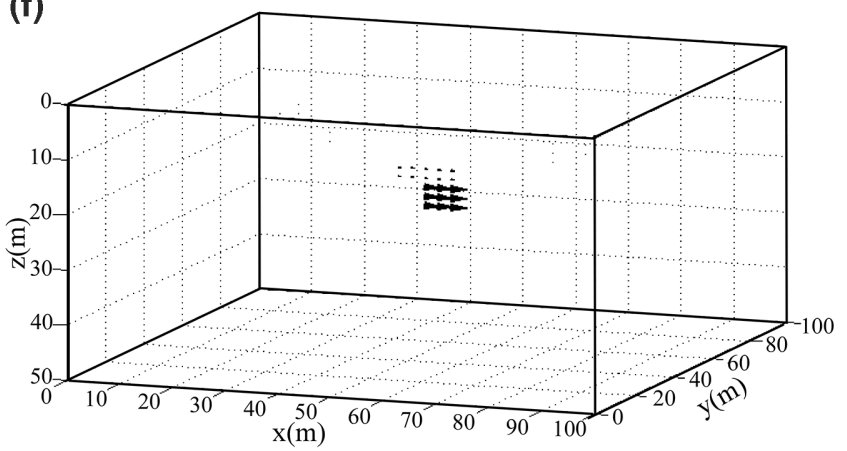

Figure 7. Simulation for the third case study with a depth of the anomaly of $25 \mathrm{~m}$. (a-c) Distribution of the three components $\left(B_{x}, B_{y}, B_{z}\right)$ of the secondary magnetic field as first case are due to a conductive block (10 Ohm m), but this time it was deeply placed at $z=25 \mathrm{~m}$ in a resistive domain (1000 Ohm). (d) This deeper heterogeneity of the electrical resistivity produces small amplitude of current sources because it moved away from the primary sources placed on the surface, (e) the magnetic signal is very low with respect to our first case however the inversion result remains satisfactory based on the shape of the anomalous current density. (f) Presentation of the vector of current density sources reconstructed from the inversion. The resulting density source is in agreement with the density corresponding to the true model.

The result of the inverse problem of this first case is reported in Fig. 5 in which the magnetic data was fitted and the main characteristics of the true model were retrieved. The algorithm successfully reconstructed both direction and magnitude of the electrical current density where the horizontal component $J_{x}$ is the principal component of the magnetic signal.

\subsection{Case study 2}

In the second case, we used the geometry described in the previous case and we changed only the contrast of the electrical resistivity between the domains by reducing the resistivity of the block to $100 \mathrm{Ohm} \mathrm{m}$ (Fig. 6). Since the amplitude of the signal depends on the contrast of the electrical resistivity, this case presents a smaller 


\section{(a)}
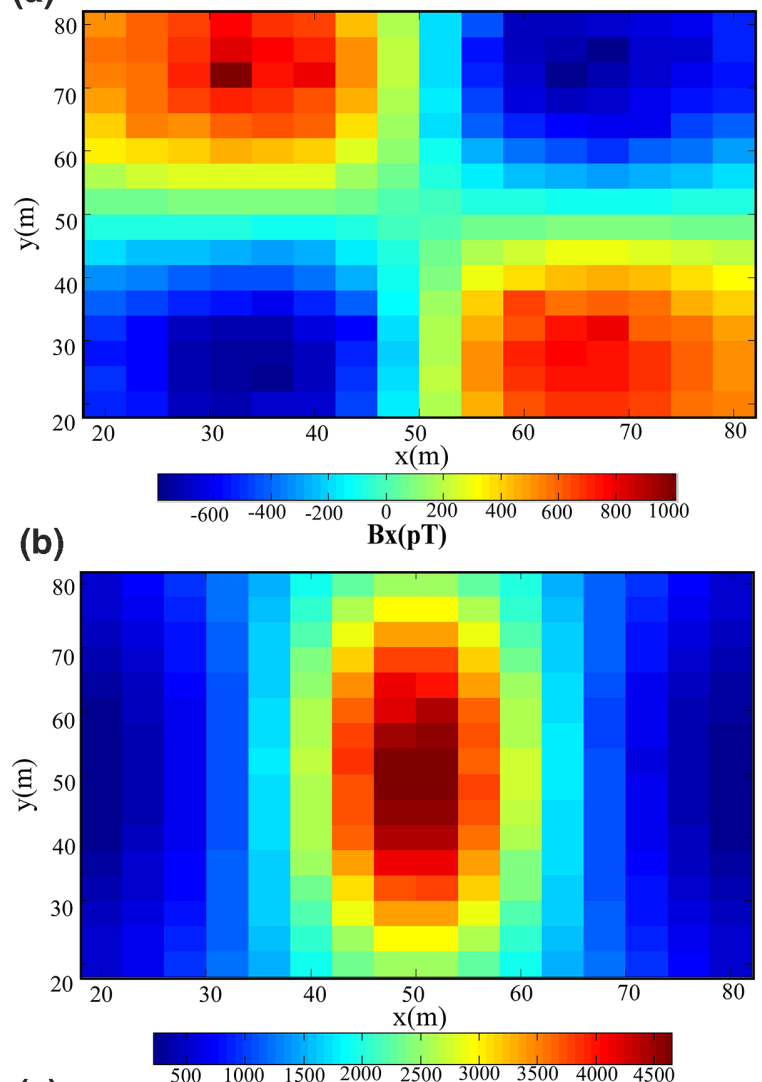

(c)

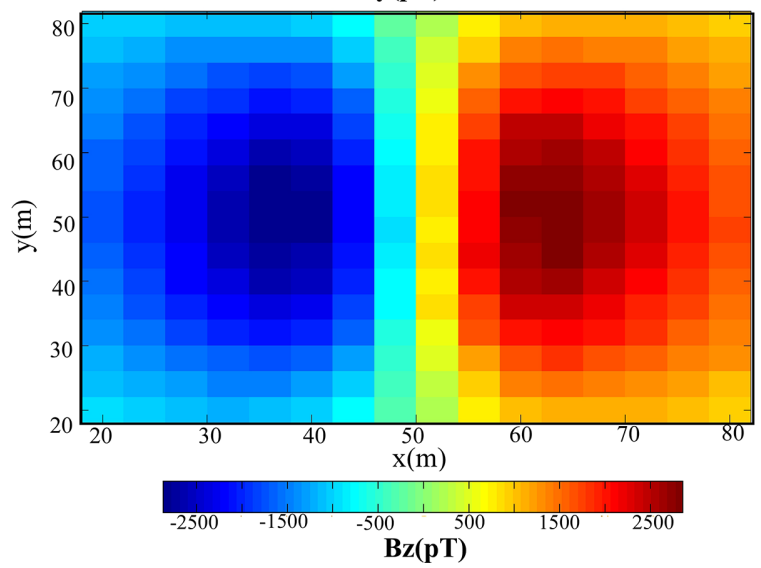

(d)

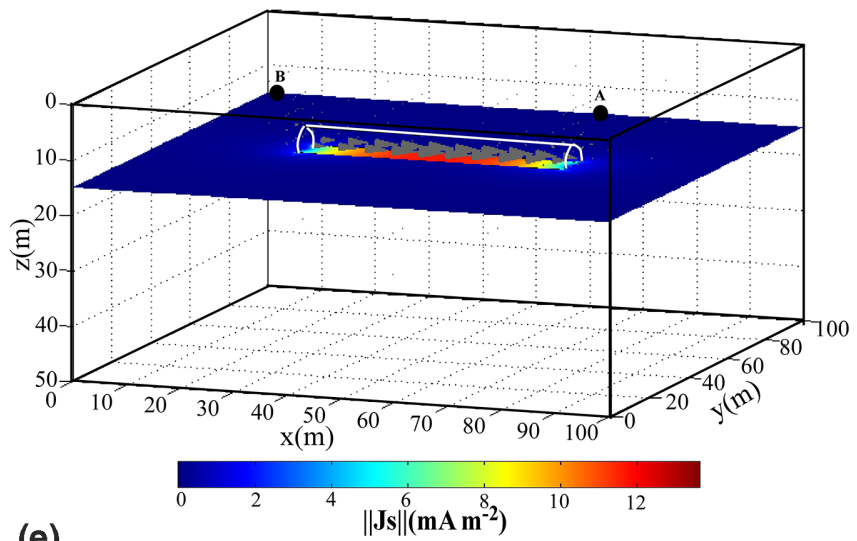

(e)

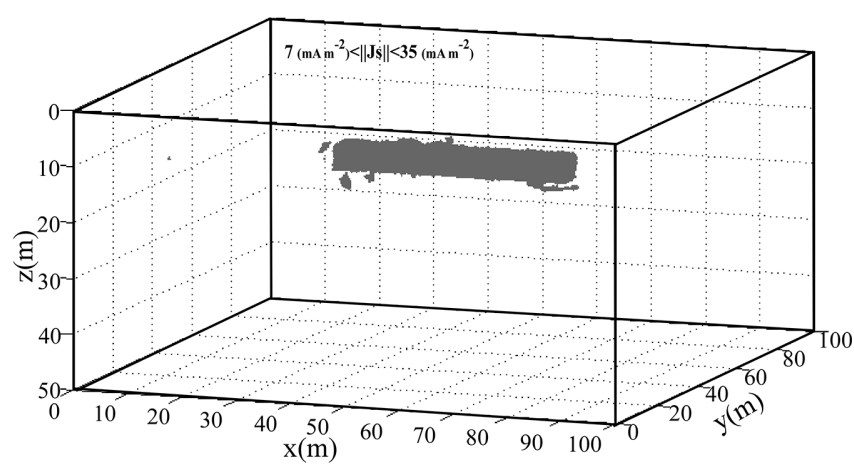

(f)

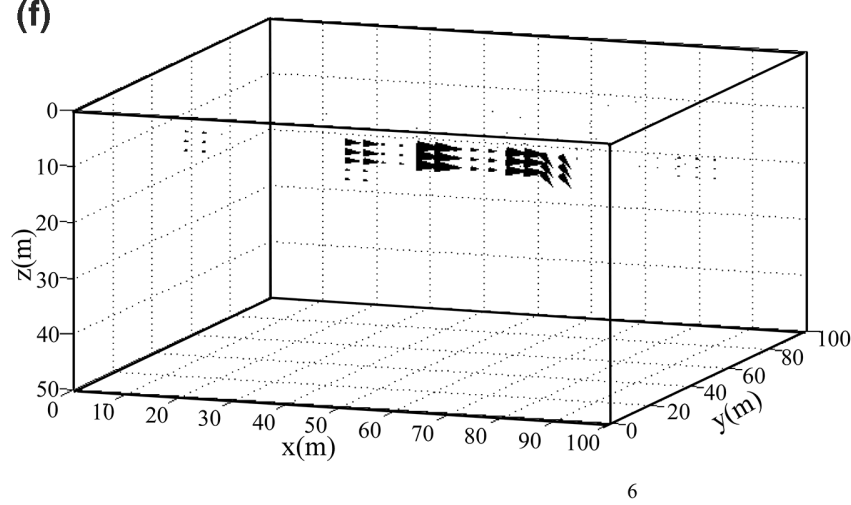

Figure 8. Simulation for the fourth case study. (a-c) Distribution of the three components $\left(B_{x}, B_{y}, B_{z}\right)$ of the secondary magnetic field are due to a heterogeneity conducted by a conductive cylinder with $10 \mathrm{Ohm} \mathrm{m}$ as resistivity buried in a resistive domain (1000 Ohm m) (Fig. 7). (d) The cylinder has a length of $50 \mathrm{~m}$ and a radius of $4 \mathrm{~m}$, and is placed at depth of $\mathrm{z}=15 \mathrm{~m}$. (e) The inversion of magnetic data allowed estimation of the spatial distribution of the current density sources that are mainly localized in the conductive cylinder. (f) Spatial distribution of the three components of current density, after inversion, presented in arrow form easily identify that the horizontal component $J_{X}$ is the principal source.

amplitude of the magnetic anomalies with respect to the previous case with a ratio of 0.54 (Fig. 6). The goal of this case is to study the ability of the approach to detect a conductive medium presenting a small contrast with respect to its background. In this case, we kept the same number of measurements and the subsurface discretization used in the precedent case to perform the inverse process.

After seven iterations, the inverse algorithm provided the inverse solution illustrated in Fig. 6 in which the conductive block was retrieved despite its small resistivity contrast to its surroundings.
The inversion succeeded well in defining the main vector of the density of the source currents which polarizes the medium which here is perfectly horizontal $\mathbf{J} x$, as shown in Fig. 6.

\subsection{Case study 3}

In this case, we used the same geometry as presented in the first case, and we only modified the depth of the block at $z=25 \mathrm{~m}$ (Fig. 7). This geometry of the anomaly and the location of the 
(a) Study case 5
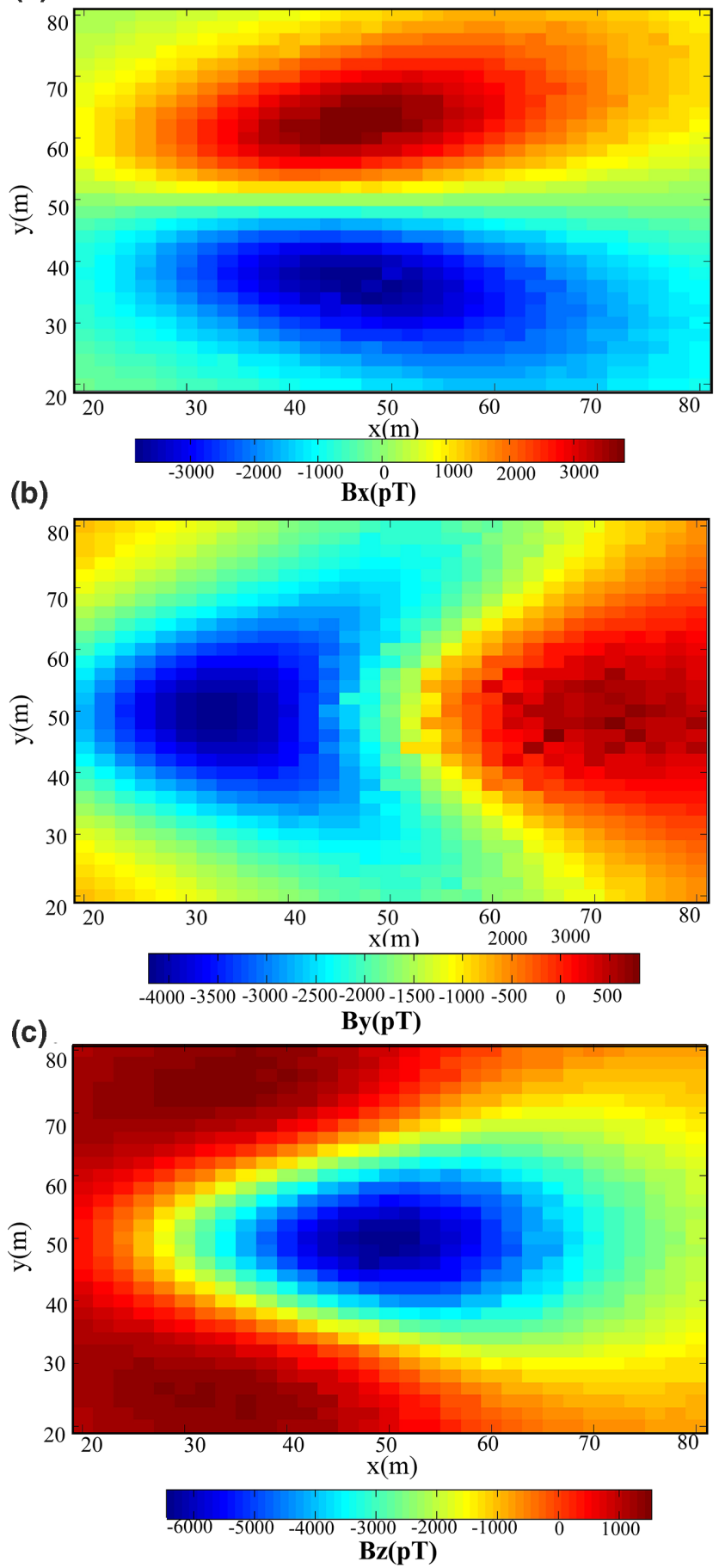

(d)

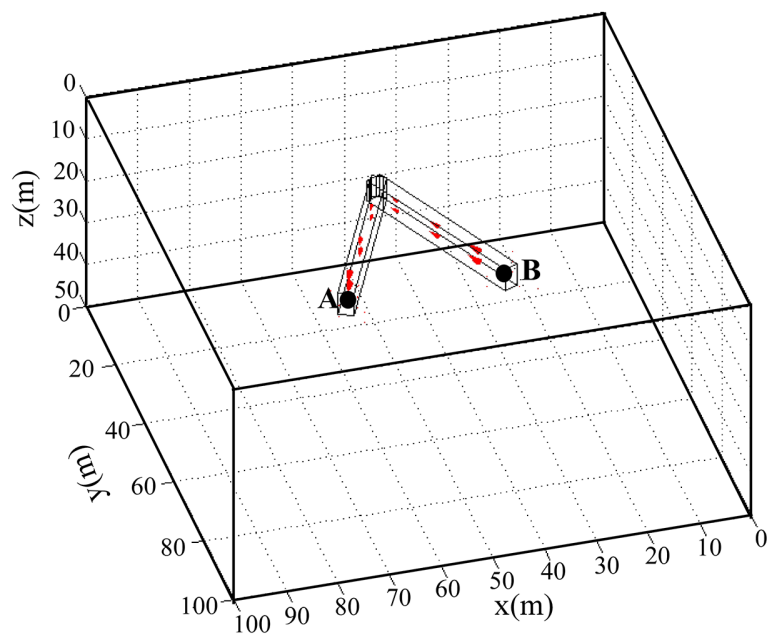

(e)

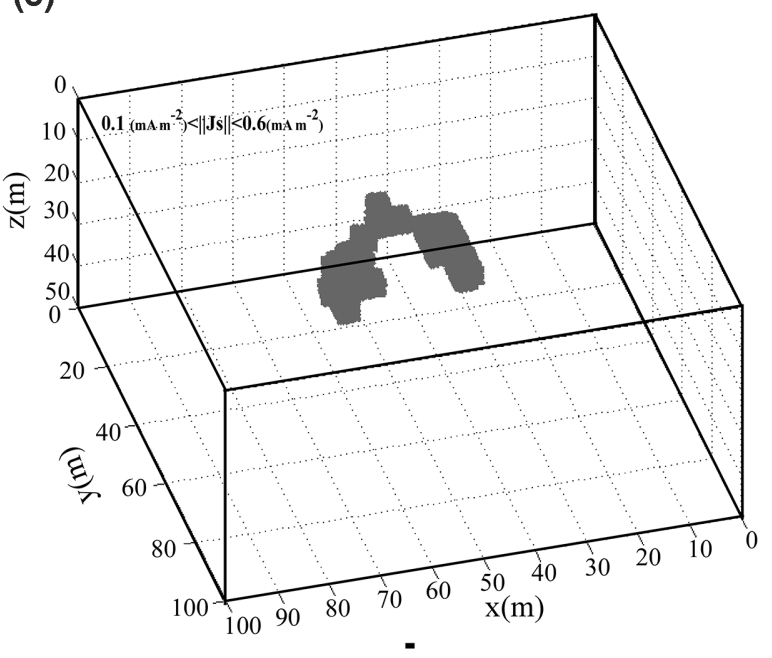

Figure 9. Simulation for the fifth case study. (a-c) Distribution of the three components $\left(B_{x}, B_{y}, B_{z}\right)$ of the secondary magnetic field are due to heterogeneity conducted by a conductive medium having $\mathrm{V}$ as shape $(10 \mathrm{Ohm} \mathrm{m})$ buried in a resistive domain $(1000 \mathrm{Ohm} \mathrm{m})$. (d) The conductive body is situated at a depth of $15 \mathrm{~m}$ and A and B denote the current electrodes. Unlike the previous case, the injection electrodes are positioned at the extremity of the conductive medium, thus the current density sources more closely follow the conductive body. (e and f) The magnetic data were successfully used to retrieve the current density source that coincides with the form of the heterogeneity as illustrated by the norm of the inverted current density.

electrodes A and B favor the appearance of a secondary horizontal current flux. The analysis of the amplitudes of magnetic anomalies makes it possible to identify the component of electrical current source associated with the observed magnetic signal. In our case the magnetic anomalies resulting from the vertical contribution of the current density can be neglected with respect to the horizontal components. Therefore, we focus on inverting only the horizontal components of the current density which permits to reduce the number of unknown parameters to estimate. The amplitude of the magnetic field corresponding to this anomaly is small compared to the first case study. However, this magnetic signature still contains the information required to locate the electrical current source as 
it shows the inversion results in the figure that was obtained after seven iterations (Fig. 7).

\subsection{Case study 4}

The secondary magnetic field is now due to a heterogeneous conductive cylinder of $10 \mathrm{Ohm} \mathrm{m}$ buried in the resistive domain characterized by a resistivity of $1000 \mathrm{Ohm} \mathrm{m}$ (Fig. 8). The cylinder has a length of $50 \mathrm{~m}$ and a radius of $4 \mathrm{~m}$, and is placed at $\mathrm{z}=15 \mathrm{~m}$. The conductive cylinder concentrates the majority of the electrical current as it was shown in the figure. The large dimension of the heterogeneity generates the magnetic anomalies with much higher amplitude (Fig. 8). These magnetic field data were recorded at $N=256$ locations distributed on surface, and the electrical current density source were discretized in $M=3072$ cells. We have performed an inversion of these data to locate this cylindrical form of heterogeneity by taking $\lambda=10^{-3}, \beta=10^{-6}$ and $\gamma=3$. The result of the inversion presented in Fig. 8(e) was obtained after four iterations, in which we can easily identify the cylindrical form of the heterogeneity. However, the nature of the compact constraint reproduced a current path with a non-uniform spatial distribution along the cylindrical geometry (Fig. 8f).

\subsection{Case study 5}

In this last case, we have generated the secondary magnetic anomalies from a heterogeneity of electrical resistivity having a $\mathrm{V}$ shape. This body is characterized by a high electrical conductivity of $10 \mathrm{Ohm} \mathrm{m}$ with respect to its environment which is very resistive $(1000 \mathrm{Ohm} \mathrm{m})$. The conductive medium has a thickness of $5 \mathrm{~m}$ and it is situated at a depth of $15 \mathrm{~m}$ (see Fig. 9). In this case, we have assumed that the heterogeneity is known in two places in which we placed the both electrodes for injecting the electric current. The electrical current follows the conductive area and produces important magnetic anomalies in the three components. These anomalies were recorded at 961 locations at the ground surface, resulting in $961 \times 3$ measurements. The inverse of this data was performed to determine the spatial distribution of vectors of the current density source defined at the 6912 cells. The inversion of three magnetic components produces a result that closely matches the complex geometry of the conductive heterogeneity (compare Figs $8 \mathrm{~d}$ and e).

\section{CASE STUDY}

\subsection{Field site}

The site test is shown in Figs 10 and 11 and is typical of the type of site described in Fig. 2. The site is composed of the mine including an open pit, a mill, a separation facility, a waste rock dump and a tailings pond. The tailings pond provides storage for tailings, waste water and drainage from the mine. Drainage through the waste rock has usually higher contaminant concentrations than the tailings water. It follows that it is preferable to collect and treat drainage from the waste rock dump before flowing into the tailings pond. The purpose of the investigation reported in this paper is used to identify preferential drainage flow paths bypassing the waste rock dump drainage collection trench to the tailings pond.

Since these flow paths are characterized by highly mineralized pore water, they are more conductive than the background porous material. By identifying preferential electric current flow paths, the MMR method has the potential to identify these drainage paths.
This requires however to place the energizing electrodes $\mathrm{A}$ and $\mathrm{B}$ in strategic positions as described in Fig. 2.

\subsection{Field survey and data reduction}

The field work took approximately two weeks to complete and was performed in 2013 and 2014. Given the length of the drainage collection trench (approximately $1900 \mathrm{~m}$ long), four separate dipoles (A, B) configurations were employed to energize different segments of the trench (Fig. 10). The four surveys are termed Survey 1 to Survey 4 . The general grid spacing was $16 \mathrm{~m} \times 16 \mathrm{~m}$, but in some locations the density of stations was increased to $8 \mathrm{~m} \times 8 \mathrm{~m}$.

To best characterize the flow paths, the energizing electrodes $\mathrm{A}$ and $\mathrm{B}$ must be strategically placed up-gradient and down-gradient of the drainage collection trench (Fig. 10). An AC electric current with a specific signature frequency $(380 \mathrm{~Hz})$ was applied to the paired $(\mathrm{A}, \mathrm{B})$ electrodes. The electric current flowing between the bipole current electrodes (A, B) generates a magnetic field at $380 \mathrm{~Hz}$. The study comprises four surveys. Survey 1 contains 607 measurement stations (the current $I$ between A and B was setup to 0.78 A). Survey 2 comprises 597 stations $(I=0.68$ A), Survey 3 is characterized by 908 stations $(I=0.75 \mathrm{~A})$, and finally Survey 4 is characterized by 497 stations $(I=0.85 \mathrm{~A})$. The investigation comprised a total of 2609 stations. At each station, the three components of the magnetic field are recorded at the ground surface in the survey area between electrodes A and B with a flux gate magnetometer. The magnetic field was used to identify the location of preferential electric current flow paths as discussed below in details. Most of the readings fell in the range of 100-1000 pT A ${ }^{-1}$ (picotesla per ampere). The median horizontal component of the field magnitude was $370 \mathrm{pT} \mathrm{A}^{-1}$. The median vertical component of the magnetic field magnitude was $128 \mathrm{pT} \mathrm{A}^{-1}$. The coil magnetometer can repeat measurements within a standard deviation of about 2 pT. The noise floor (mean ambient field noise, determined from a sampling of several frequencies in the spectrum) remained low (around $6 \mathrm{pT}$ ) and constant throughout the investigation. Numerous measurements were repeated throughout the course of the fieldwork to ensure consistent, repeatable data.

Along the toe of the waste rock dump, conductive culture associated with a buried power line was present. The 7 th harmonic of the $60 \mathrm{~Hz}$ power was detected and mapped by the Willowstick instrument (detected at $420 \mathrm{~Hz}$ ) with magnitudes in the range of 10-200 picotesla. The presence of this power line also caused some magnetic interference at $380 \mathrm{~Hz}$ due to electrical currents from the $380 \mathrm{~Hz}$ electric source signal that followed the power line. Such can locally interfere with the interpretation of the magnetic signal from the subsurface directly beneath the conductive culture. Fortunately, the location of conductive culture was known and was taken into account during the modelling and interpretation of the data.

Only Survey 1 is presented in detail below. Survey 1 targets preferential groundwater flow paths through the north end of the drainage collection trench. Since the other three surveys use the same data collection, interpretation and modelling process, we focus only on this survey in this paper and we will show the general result for the four surveys in the last part of this section. Fig. 12 presents the current electrodes A and B position and survey layout for Survey 1 .

Fig. 13 presents the observed magnetic field contour map created from the injected electric current through Survey 1's study area. Some areas are influenced by cultural noise (Fig. 13). In order to 


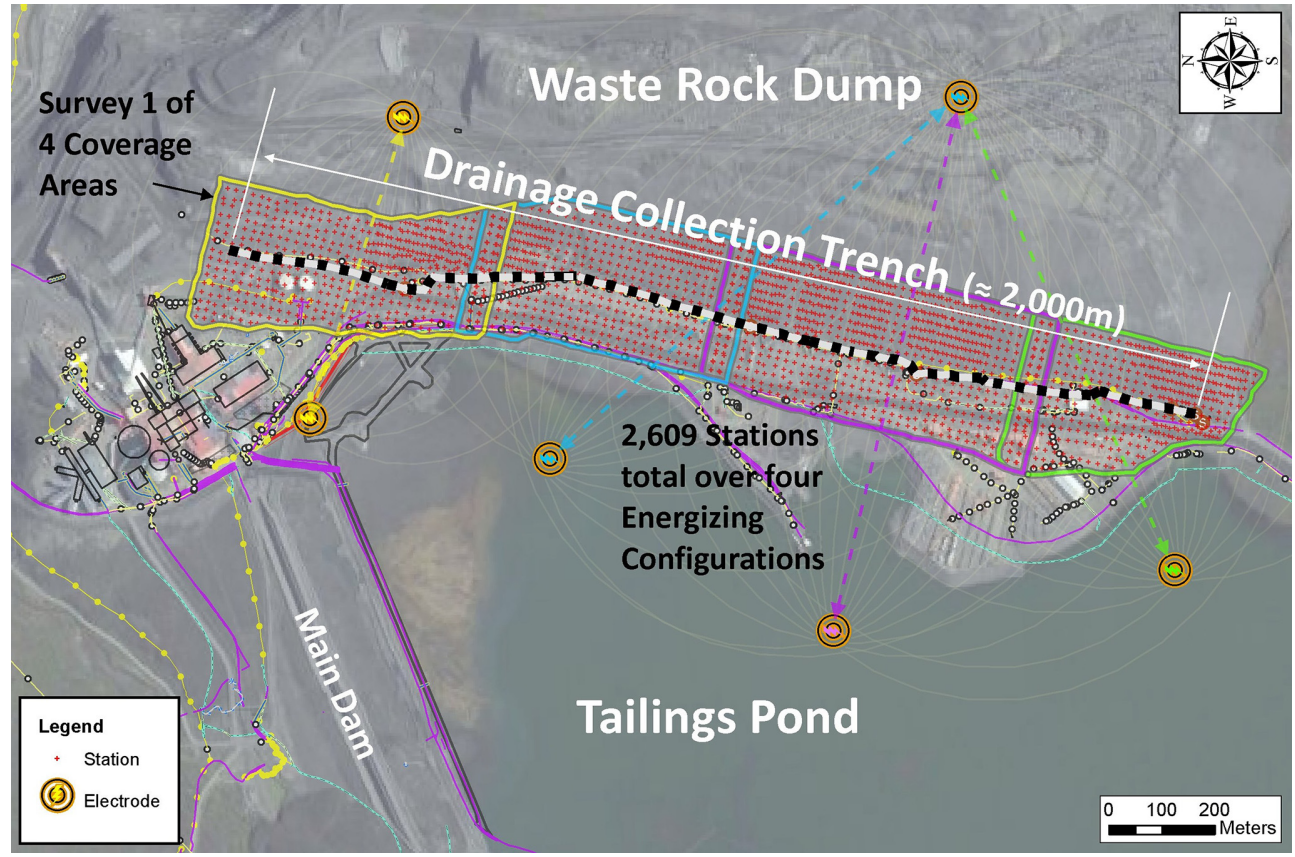

Figure 10. Test site. Position of the waste rock dump and tailings pond with drainage collection trench between (dotted black and white line). Note the presence of a buried powerlines (yellow lines with yellow dots) crossing the survey areas. The investigated area is divided into 4 sub-surveys S1 to S4.

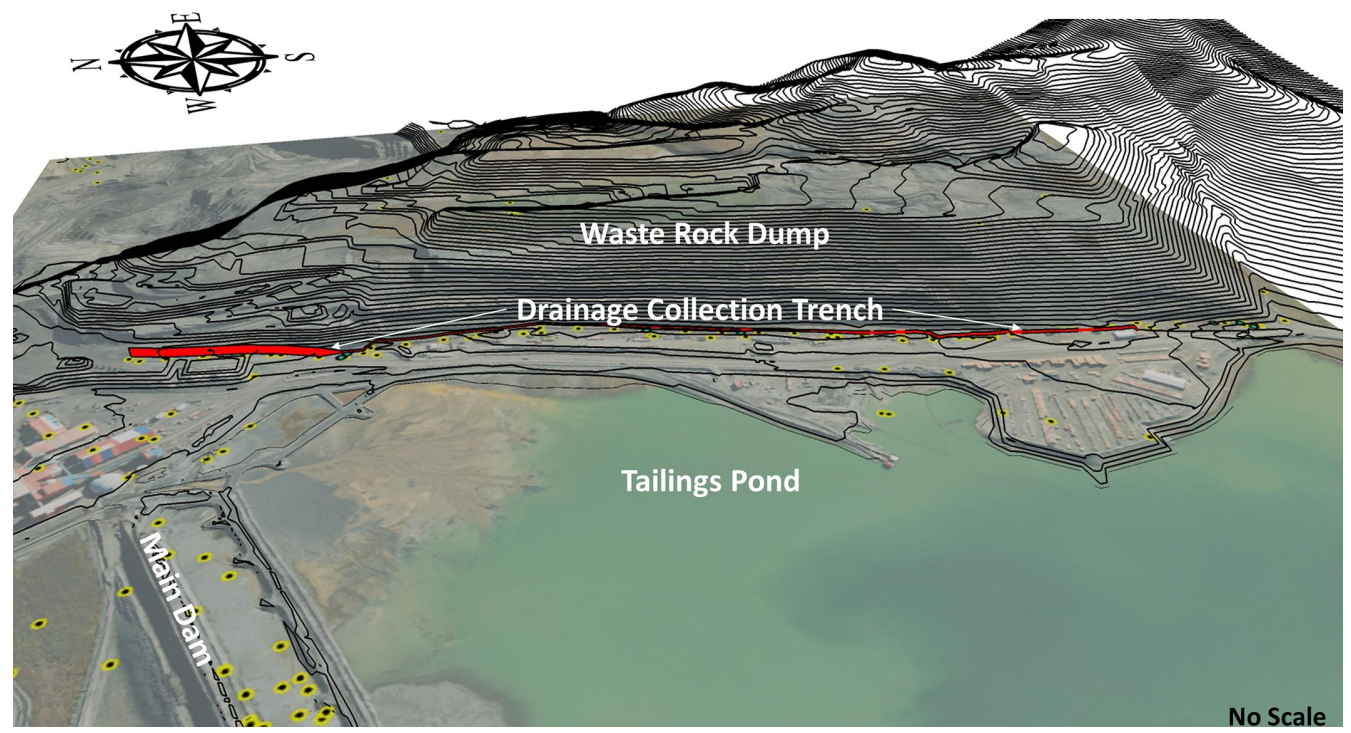

Figure 11. Topography of the survey area (looking East from above) showing the position of the drainage collection trench at the bottom of the waste rock dump.

provide the very best interpretation of the data as possible, measurement stations influenced by conductive culture were filtered out before modelling or interpretation. The recorded magnetic field must also be corrected using the predicted magnetic field model based on a current distribution in an electrically homogeneous subsurface. This causes the concentration of electric current due to changes in subsurface conductivity (such as preferential groundwater flow paths) to stand out much better. A flow current model was therefore created to predict the magnetic field response (per Amp) expected at each measurement station given the position of the circuit wire, the electrodes (A, B), the topography of the area, and a homogenous subsurface conductivity environment. For the case of the Survey 1 energizing configuration, the forward model to predict the magnetic field used 81225 cells. The horizontal magnitude of the predicted magnetic field is represented in Fig. 14. By dividing the observed magnetic field map (Fig. 13) by the predicted magnetic field map (Fig. 14), a ratio response map is created which corrects for the electrode effect and shows areas of anomalous electric current flow-greater or lesser than predicted. The result is shown in Fig. 15.

In Fig. 15, the white shaded contours (where the ratio is approximately $1: 1$ ) show where the magnetic field intensity is equivalent to that predicted by the homogeneous model. Areas shaded purple indicate magnetic field is less than predicted, and areas shaded green indicate magnetic field is greater than predicted. Preferential flow paths are revealed more by the shape of contours than by the color of 


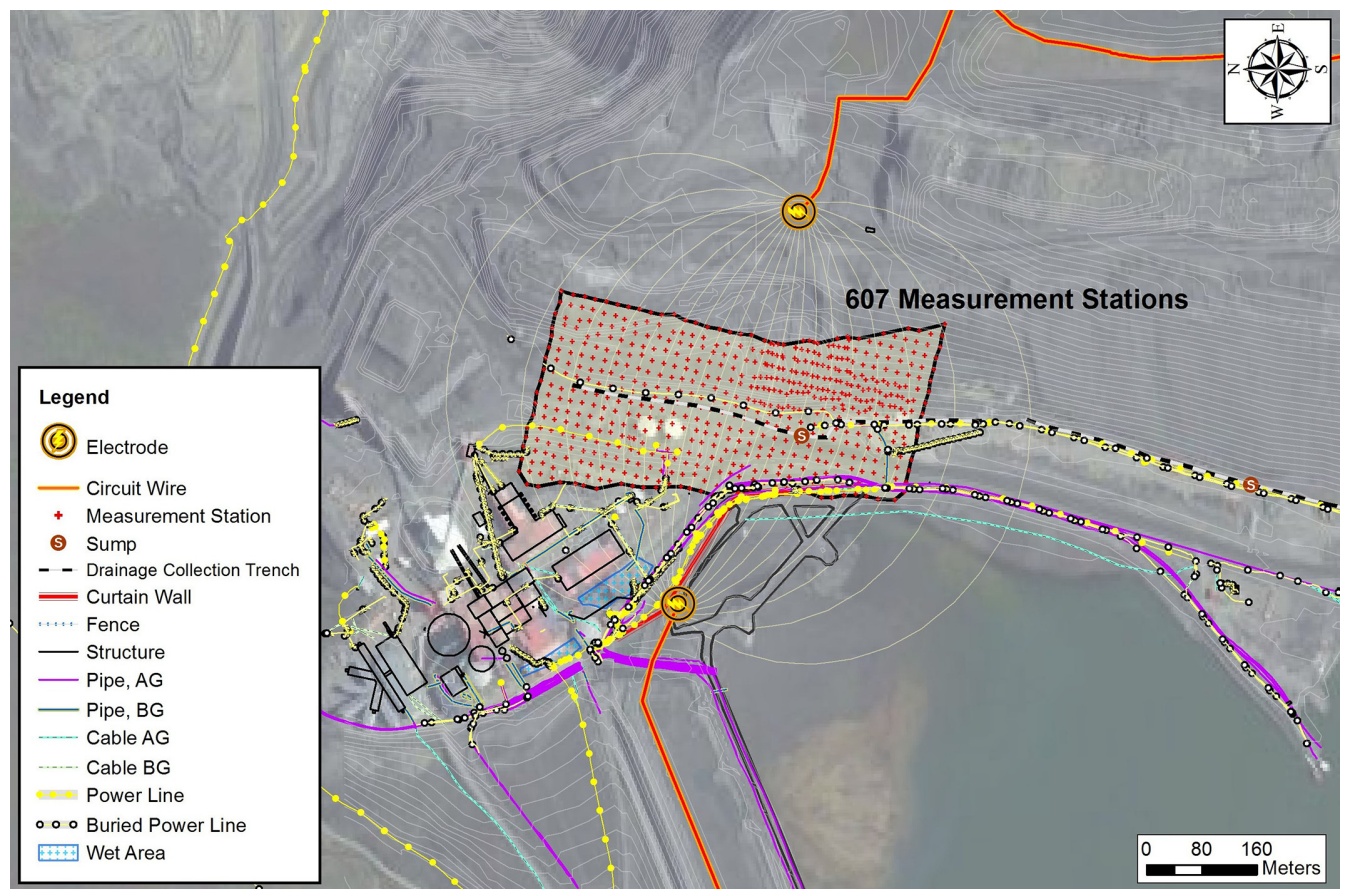

Figure 12. Survey 1 showing the position of the energizing electrodes A and B and the stations where the three components of the magnetic field are measured. The small red symbols denote the measurement stations (536 stations, after filtering, are considered in this survey). The measurement stations were approximately established on a $16.5 \mathrm{~m}$ grid and localized with a differential GPS. Some measurement stations were occupied repeatedly for quality control purposes. The up-gradient electrode was placed in a well completed in the waste rock dump and in contact with groundwater flowing through and/or beneath the pile. The down-gradient electrode was placed in the tailings pond itself. The red/orange circuit wire connecting the current electrodes A and B was positioned in a large loop around the study area. The electrodes and circuit wire are located outside the study area as much as possible due to the strong magnetic field B0 generated by the cable. The thin yellow lines are representative of the general distribution of electric current for a homogeneous half-space.

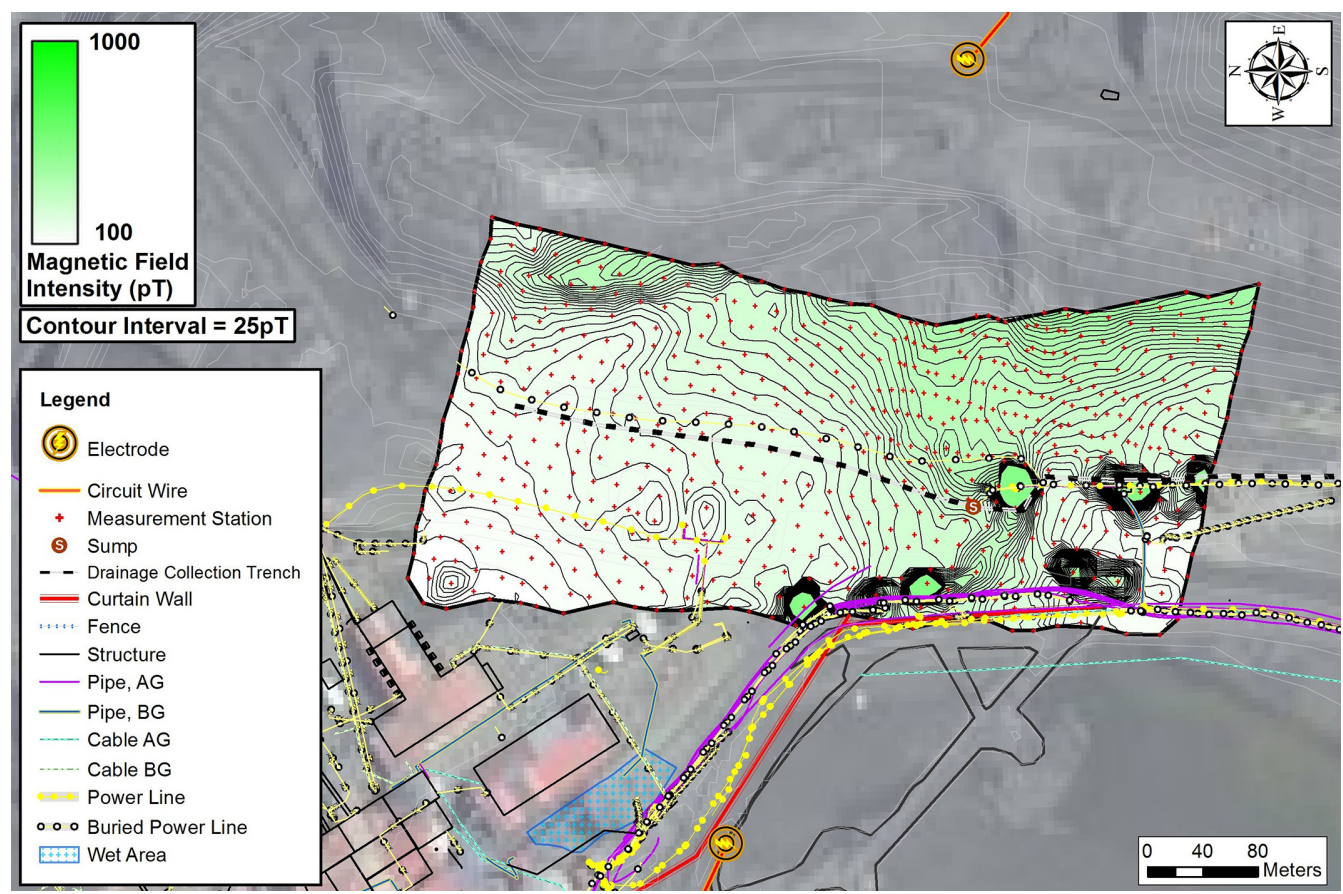

Figure 13. Observed magnetic field contour map. In some locations, electric current follows along conductive culture as evident by the steep gradient of the magnetic field (appearing as dense black lines in the figure). In order to interpret the data from these recorded data, these corrupted stations are removed before analysing the magnetic field map (see the x-symbols in Fig. 15). 


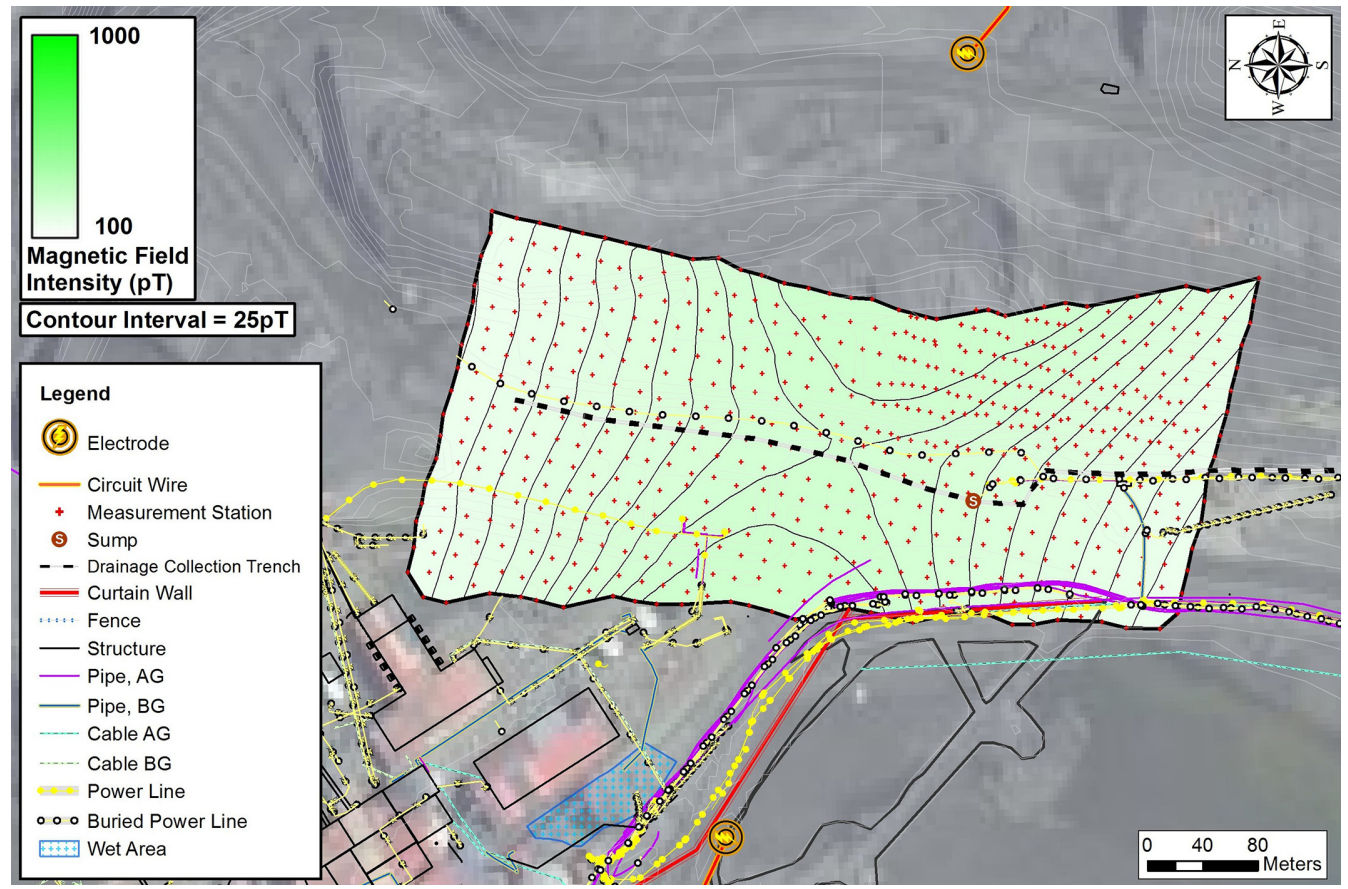

Figure 14. Predicted magnetic field contour map for a homogeneous conductive subsurface. The magnetic field is computed by simulating the dc- current flowing in the subsurface between the two current electrodes shown in the figure.

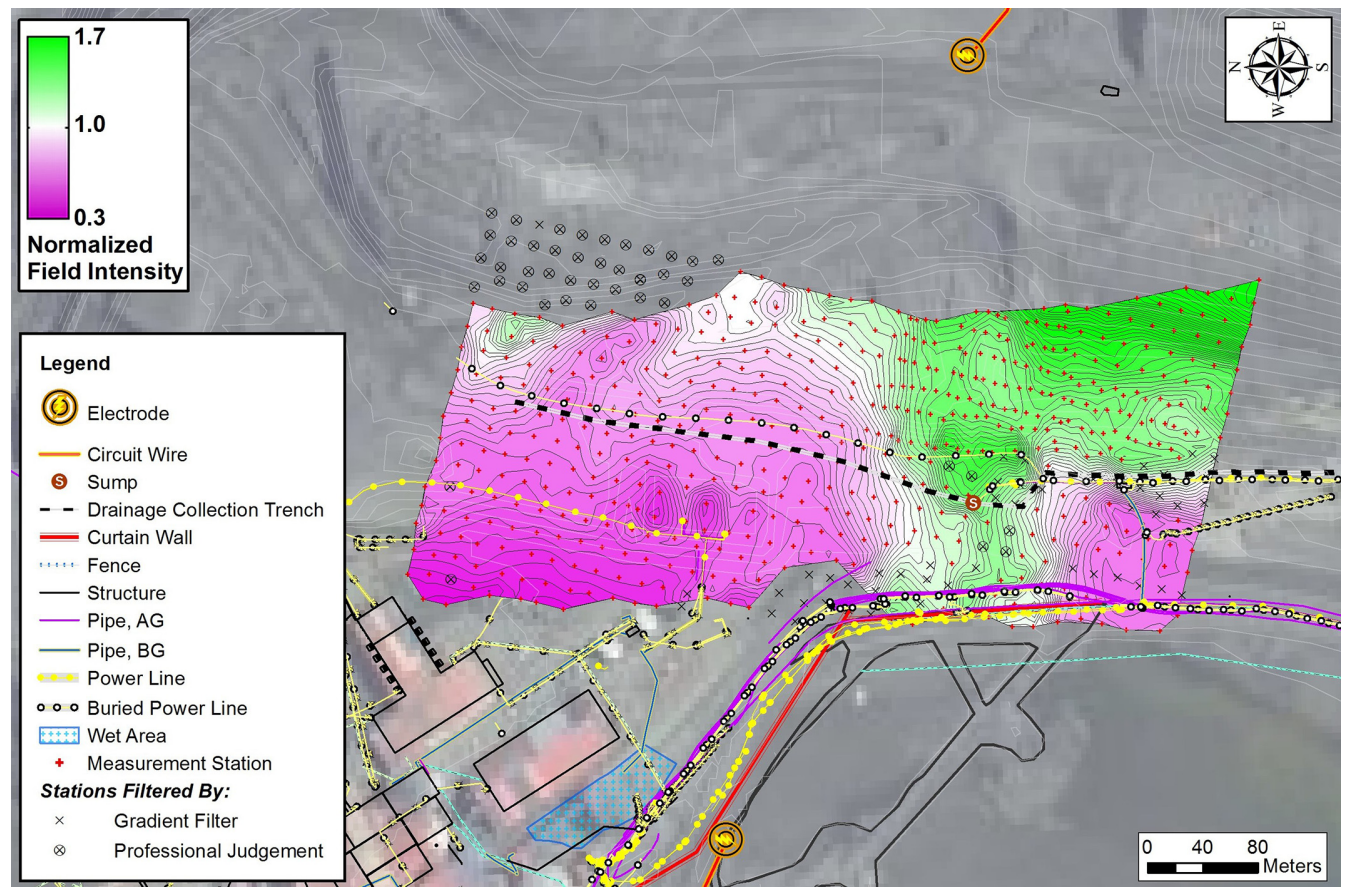

Figure 15. Ratio response contour map. This is the map that is inverted to determine the 3-D distribution of the focused current in the subsurface. Measurement stations removed from the data set are shown as black ' $x$ ' symbols (gradient filter) or circled ' $x$ ' symbols (professional judgement) in this figure.

shading in any given area. Next the ratio response map is inverted to determine the distribution of the electric current flow in 3-D. The inversion model domain for Survey 1 covered $608 \mathrm{~m} \times 392 \mathrm{~m} \times 147 \mathrm{~m}$ with a discretization of cells $8 \mathrm{~m} \times 8 \mathrm{~m} \times 3 \mathrm{~m}$ in size. There were 182476 cells in the mesh and 120246 of these were active during the iterative inversion process.

Fig. 16 shows the electrical current distribution associated with the inversion of the ratio response map. Fig. 16 presents a horizontal slice (plan view) of the electrical current distribution model taken at elevation $280 \mathrm{~m}$. For reference, the ground elevation at the toe of the waste dump is approximately $305 \mathrm{~m}$.

In Fig. 16, the white to dark green shading (going up the scale) signifies increasing levels of anomalous electric current density. The light blue to purple shading (going down in scale) signifies decreasing levels of electric current density anomaly. The yellow arrows identify preferential flow paths that electric current follows 


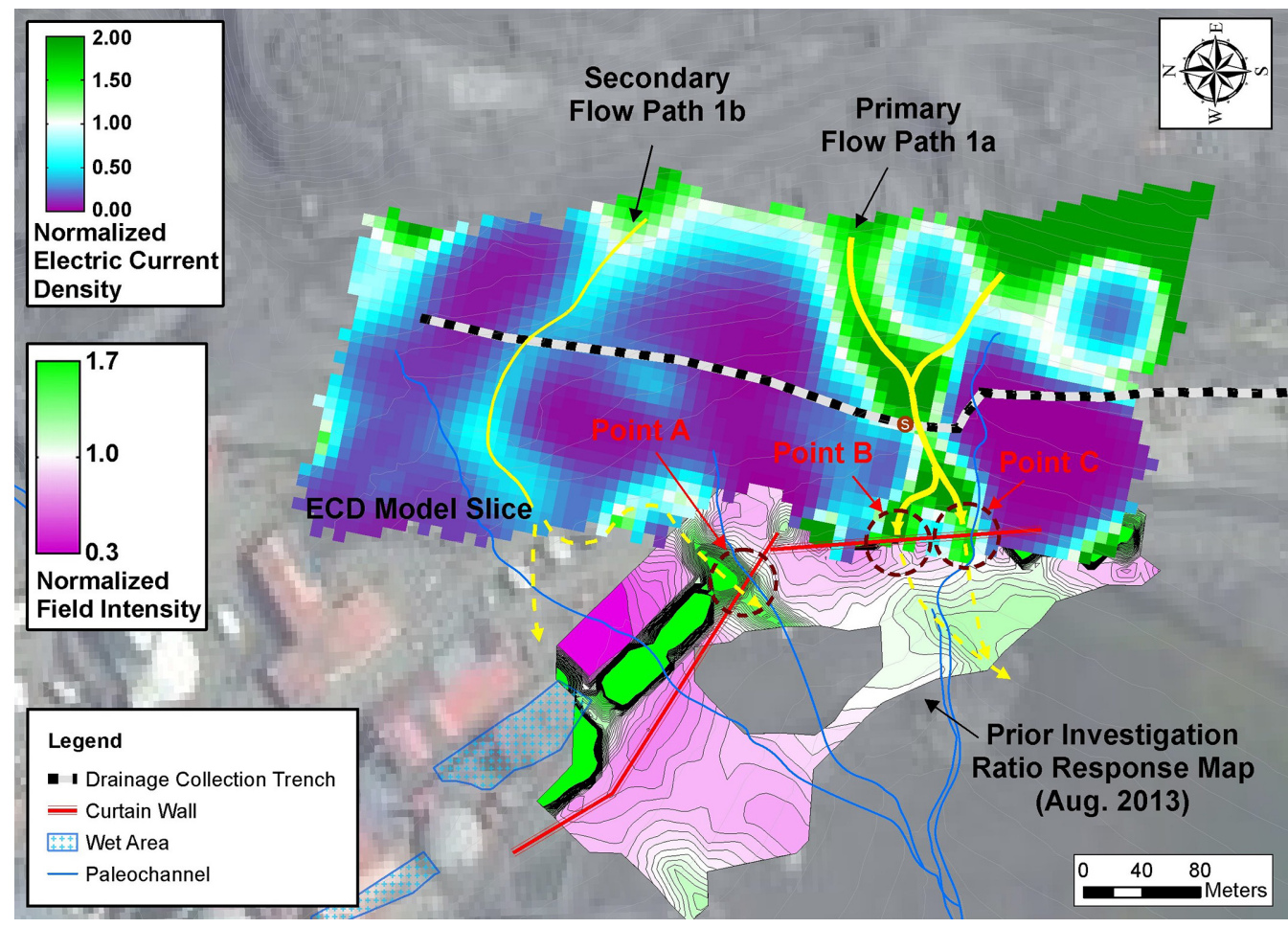

Figure 16. Current flow path distribution at an altitude of $280 \mathrm{~m}$. The blue lines correspond to broad and deep erosional channel based on pre-mining topographic contours.

through Survey 1. Two flow paths were identified where electric current passes through or beneath the drainage collection trench in two locations. Both paths are believed to convey contaminated water toward the tailings pond. The two flow paths have been labeled 1a and $1 \mathrm{~b}$ in reference to the survey in which they were found. Flow paths are also classified as primary or secondary based on the strength of the anomalous electric current intensity. This classification is also used to suggest that more water flows through a primary flow path than a secondary flow path. Primary Flow Path 1a was found to align with a pre-mining drainage channel, most easily identified as a broad and deep erosional channel based on pre-mining contours. This flow path extends from the bottom of the drainage collection trench to well beneath the trench. The secondary flow path appears to flow just beneath the drainage collection trench but at a much shallower depth than the primary flow path. Note that although the slice at $280 \mathrm{~m}$ (Fig. 16) shows the footprint of both flow paths, the estimated elevations of each flow path was somewhat different and was not necessarily at $280 \mathrm{~m}$ elevation.

\subsection{Final result}

Using the previous methodology, all the interpreted flow paths are shown in Fig. 17. In order to rank them in terms of perceived importance, they are classified as primary or secondary flow paths based on the anomalous electric current strength. This nomenclature is used to suggest that more water may flow through a primary flow path than a secondary. As shown in Fig. 17, primary flow paths were found to align with pre-mining drainage channels (shown by blue lines in Fig. 17). They are identified as broad and deep erosional channels based on pre-mining topographic contours. All secondary flow paths appear to flow beneath the drainage collection trench but at generally shallower depths than the primary flow paths. In almost all cases, the tops of the flow paths intersect the bottom of the drainage collection trench. This type of information can be used to take effective decisions concerning how to further monitor and remediate ground water bypassing the waste rock dump drainage collection trench into the tailings pond.

\section{DISCUSSION}

As shown in the previous sections, MMR can be used to determine conductive pathways, which can be interpreted, under specific circumstances, to preferential flow paths of groundwater. These conductive flow paths can be hardly detected using other techniques. Electrical resistivity alone is rarely a good detector of the permeability since, depending on the clay content, positive and negative correlations exist between resistivity and permeability (Purvance $\&$ Andricevic 2000a,b). In addition, electrical resistivity tomography is limited by its lack of resolution. Induced polarization is sensitive to permeability (e.g. Binley et al. 2005; Revil et al. 2015) but suffer from the same limitations than electrical resistivity tomography regarding its resolution.

Another point worth to be discussed is the fact that MMR does not require a static map of the magnetic field. Such map is indeed irrelevant since we are only interested by the magnetic field produced by the injected current at a given frequency. The use of a three components flux gate magnetometer that works in a narrow frequency band around the frequency of the injected current can be used to filter out other magnetic sources, especially the static component. In addition, one may wonder about the validity of the quasi-static approximation used in this paper while we are using a $380 \mathrm{~Hz}$ electrical current source. In this paper, we have neglected any induction effect. We will need however to check in future publications if this assumption is always valid, especially for very conductive environments. 


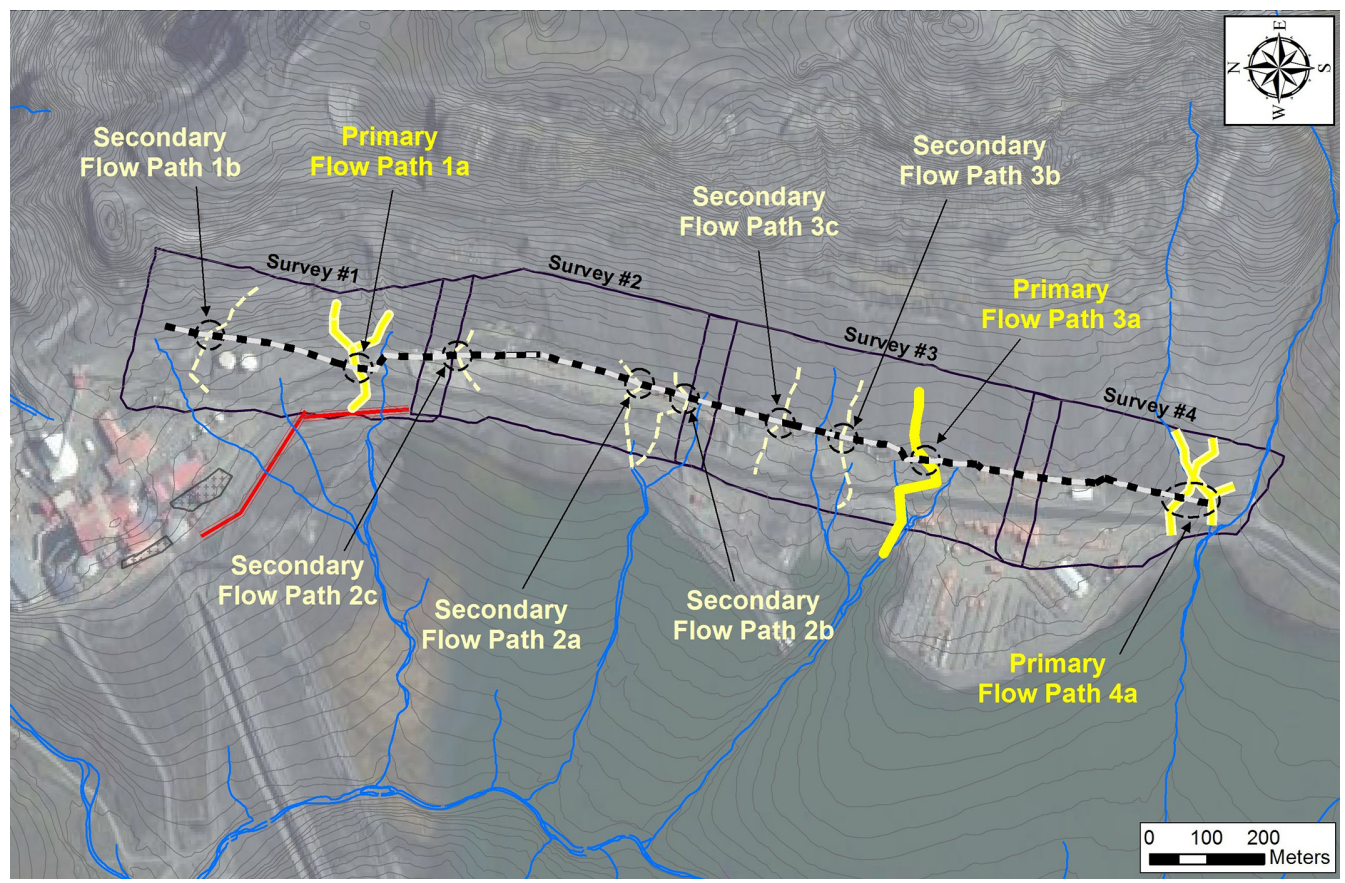

Figure 17. Summary of the two types of flow paths that have been identified by the MMR survey. The network of blue lines corresponds to pre-mining drainage channels identified as broad and deep erosional channels based on pre-mining topographic contours.

Other methods can be used to detect conductive paths in addition to MMR. For instance the mise-à-la masse technique has been extensively used to detect preferential flow paths in a variety of environments (Ramirez et al. 1996; Daily et al. 2004). That said, this technique works well when we know where the conductive paths start or end, and it has the advantage of taking measurements in free space, without requiring galvanic contact with the ground. For the type of application described in this paper, it is difficult to know if the mise-à-la masse technique would present any advantages with respect to the MMR technique. This wil constitute an interesting researc topic in itself.

The self-potential method offers also a technique that can be used to detect preferential flow paths (e.g. Bolève et al. 2009; Ikard \& Revil 2014). This technique is based on the fact that preferential flow paths generate their own electrical current due to streaming potential phenomena. Flowpaths are characterized by slighly more negative electrical potential values. With the recent developments of inversion techniques able to use self-potential signals to invert permeability, this technique can be used to provide 3-D images of preferential flow paths in 3-D. However, the self-potential signals are sensitive to the mineralization of the pore water and highly mineralized ground water implies low self-potential signals. Therefore the self-potential technique could not be used to detect preferential flow paths in the present context.

\section{CONCLUSIONS}

Conductive paths can be detected through the MMR method. The equation for the secondary magnetic field associated with the injection of a current between two current electrodes for a subsurface with a conductive target takes the form of the Biot and Savart law. This equation is linear between the resulting magnetic field and the causative conduction current density. This equation is used to define a kernel of analytical Green's functions. We then recast the inverse problem as a potential field problem between the secondary magnetic field and the conduction current. The solution of this problem is rather straightforward and we use a classical deterministic approaches used to invert potential field data. The methodology is benchmarked using a set of five synthetic case studies with targets of different shapes, sizes, depths and resistivity contrasts between the anomaly (i.e., the target) and the background. We were successfully able to replicate the position of the target and its shape for these five case studies. A successful field application is performed to the detection of electrically conductive flow paths (containing highly mineralized pore waters) bypassing a drainage collection trench between a waste rock dump and tailings pond.

\section{ACKNOWLEDGEMENTS}

This work is funded by Willowstick Technologies, LLC. We thank Nicolas Florsch and an anonymous referee for their very constructive comments and the Editor for handling this manuscript. The work of A. Revil is also funded by RESBA ALCOTRA.

\section{REFERENCES}

Acosta, J.E. \& Worthington, M.H., 1983. A borehole magnetometric resistivity experiment, Geophys. Prospect., 31, 800-809.

Binley, A., Hubbard, S.S., Huisman, J.A., Revil, A., Robinson, D.A., Singha, K. \& Slater, L.D., 2015. The emergence of hydrogeophysics for improved understanding of subsurface processes over multiple scales, Water Resour. Res., 51, 3837-3866.

Binley, A., Slater, L.D., Fukes, M. \& Cassiani, G., 2005. Relationship between spectral induced polarization and hydraulic properties of saturated and unsaturated sandstone, Water Resour. Res., 41, W12417, doi:10.1029/2005WR004202.

Bolève, A., Revil, A., Janod, F., Mattiuzzo, J.L. \& Fry, J.-J., 2009. Preferential fluid flow pathways in embankment dams imaged by self-potential tomography, Near Surf. Geophys., 7(5), 447-462. 
Chen, J., Haber, E. \& Oldenburg, D.W., 2002. Three-dimensional numerical modelling and inversion of magnetometric resistivity data, Geophys. J. Int., 149, 679-697.

Daily, W., Ramirez, A. \& Binley, A., 2004. Remote monitoring of leaks in storage tanks using electrical resistance tomography: application at the hanford site, J. Environ. Eng. Geophys., 9(1), 11-24.

Dey, A. \& Morrison, H.F., 1979. Resistivity modeling for arbitrarily shaped two-dimensional structures, Geophys. Prospect., 27, 106-136.

Edwards, R.N., 1974. The magnetometric resistivity method and its application to the mapping of a fault, in Canadian Journal of Earth Sciences, 11, pp 1136-1156, NRC Research Press.

Edwards, R.N. \& Howel, E.C., 1976. Field test of the magnetometric resistivity (MMR) method, Geophysics, 41, 1170-1183.

Hansen, P.C., 1998. Rank-Deficient and Discrete Ill-Posed Problems: Numerical Aspects of Linear Inversion, pp. 247, Soc. for Industrial and Applied Mathematics.

Ikard, S.J. \& Revil, A., 2014. Self-potential monitoring of a thermal pulse advecting through a preferential flow path, J. Hydrol., 519(A), 34-49.

Jakosky, J.J., 1940. Exploration Geophysics, Times-Mirror Press.

Jakosky, J.J. 1933. Method and Apparatus for Determining Underground Structure, US Patent No. 1906271

Jardani, A., Revil, A., Bolève, A. \& Dupont, J.P., 2008. 3D inversion of self-potential data used to constrain the pattern of ground water flow in geothermal fields, Journal of Geophysical Research, 113, B09204, doi: 10.1029/2007JB005302.

Johnson, D.L., Plona, T.J. \& Kojima, H., 1986. Probing porous media with 1st sound, 2nd sound, 4th sound and 3rd sound, in Physics and Chemistry of Porous Media, Vol. 2, pp. 243-277, eds Jayanth, R., Banavar, J. \& Winkler, K. W., Am. Inst. Phys.

Kemna, A., Vanderborght, J., Kulessa, B. \& Vereecken, H., 2002. Imaging and characterisation of subsurface solute transport using electrical resistivity tomography (ERT) and equivalent transport models, J. Hydrol., 267, 125-146.

Kulessa, B., Jaekel, U., Kemna, A. \& Vereecken, H., 2002. Magnetometric resistivity (MMR) imaging of subsurface solute flow: Inversion framework and laboratory tests, J. Environ. Eng. Geophys., 7(3), 111-118.

Labrecque, D., Casale, D., Heath, G.L. \& Svoboda, J.M., 2002. Combined electrical and magnetic resistivity tomography: Theory and inverse modeling, Proceedings of the SAGEEP, EEGS, Las Vegas.

Labreque, D., Sharpe, R., Casale, D., Heath, G.L. \& Svoboda, J.M., 2003. Combined electrical and magnetic resistivity tomography: synthetic model study and inverse modeling, J. Environ. Eng. Geophys., 8, 251-262.

Last, B.J. \& Kubik, K., 1983. Compact gravity inversion, Geophysics, 48(6), 713-721.

Levy, S., Adam, D. \& Bresler, Y., 2002. Electromagnetic impedance tomography (EMIT): a new method for impedance imaging, in IEEE Transactions of Medical Imaging, 21, pp 676-687, IEEE.
Li, Y. \& Oldenburg, D.W., 1996. 3-D inversion of magnetic data, Geophysics, 61(2), 394-408.

Li, Y. \& Oldenburg, D.W., 1998. 3-D inversion of gravity data, Geophysics, 63(1), 109-119.

Li, Y. \& Oldenburg, D.W., 2000. Joint inversion of surface and threecomponent borehole magnetic data, Geophysics, 65(2), 540-552.

Nabighian, M.N., 1991a. Investigations in Geophysics no. 3: Electromagnetic Methods in Applied Geophysics, V ol. 1, Theory. SEG, Tulsa.

Nabighian, M.N., 1991b. Investigations in Geophysics no. 3, Electromagnetic Methods in Applied Geophysics, Vol. 2, Application, part A and B. SEG, Tulsa.

Nabighian, M.N., Oppliger, G.L., Edwards, R.N., Lo, B.B.H. \& Cheesman, S.J., 1984. Cross-hole magnetometric resistivity (MMR), Geophysics, 49, 1313-1326.

Pedersen, L., 1977. Interpretation of potential field data: a generalized inverse approach, Geophys. Prospect., 25, 199-230.

Purvance, D.T. \& Andricevic, R., 2000a. On the electrical-hydraulic conductivity correlations in aquifers, Water Resour. Res., 36(10), 2905-2914.

Purvance, D.T. \& Andricevic, R., 2000b. Geoelectric characterization of the hydraulic conductivity field and its spatial strcture at variable scales, Water Resour. Res., 36(10), 2915-2925.

Ramirez, A., Daily, W., Binley, A., LaBrecque, D. \& Roelant, D. 1996. Detection of leaks in underground storage tanks using electrical resistance methods, J. Environ. Eng. Geophys., 1(3), 189-203.

Revil, A., Binley, A., Mejus, L. \& Kessouri, P., 2015. Predicting permeability from the characteristic relaxation time and intrinsic formation factor of complex conductivity spectra, Water Resour. Res., 51, doi:10.1002/2015WR017074.

Revil, A., Karaoulis, M., Johnson, T. \& Kemna, A., 2012. Review: some lowfrequency electrical methods for subsurface characterization and monitoring in hydrogeology, Hydrogeol. J., 20(4), 617-658.

Stefanescu, S. \& Nabighian, M., 1962. Uber magnetische storfelder als folge senkrechter schichtungen im gleischstrom, Revue de Géologie et de Géographie, Académie de la République Populaire Roumaine, 6, 139155 .

Svoboda, J.M., Canan, B., Morrison, J.L., Heath, G.L. \& Labrecque, D., 2002. Advanced technology for mapping subsurface water conductivity, Proceedings of SAGEEP 2002, EEGS, Las Vegas.

Waxman, M.H. \& Smits, L.J.M., 1968. Electrical conductivities in oil bearing shaly sands, SPE J., 8, 107-122.

Yaramanci, U., Kemna, A. \& Vereecken, H., 2005. Emerging technologies in hydrogeophysics, Hydrogeophysics, pp. 467-486, Springer.

Zhdanov, M. \& Tolstaya, E., 2004. Minimum support nonlinear parametrization in the solution of a 3D magnetotelluric inverse problem, Inverse Problems, 20(3), 937.

Zhu, K. \& Yang, J., 2008. Time-dependent magnetometric resistivity anomalies of groundwater contamination: synthetic results from computational hydro-geophysical modeling, Appl. Geophys., 5(4), 322-330. 